\title{
Observational determination of squeezing in relic gravitational waves and primordial density perturbations
}

\author{
Sukanta Bose* \\ Department of Physics and Astronomy, P. O. Box 913, Cardiff University, CF24 3YB, United Kingdom \\ and Max Planck Institut für Gravitationsphysik, Albert Einstein Institut, Am Mühlenberg 1, Golm, D-14476, Germany \\ L. P. Grishchuk \\ Department of Physics and Astronomy, P. O. Box. 913, Cardiff University, CF24 3YB, United Kingdom \\ and Sternberg Astronomical Institute, Moscow University, Moscow 119899, Russia
}

(Received 29 November 2001; published 29 August 2002)

\begin{abstract}
We develop a theory in which relic gravitational waves and primordial density perturbations are generated by strong variable gravitational field of the early Universe. The generating mechanism is the superadiabatic (parametric) amplification of the zero-point quantum oscillations. The generated fields have specific statistical properties of squeezed vacuum quantum states. Macroscopically, squeezing manifests itself in a nonstationary character of variances and correlation functions of the fields, the periodic structures of the metric power spectra, and, as a consequence, in the oscillatory behavior of the higher order multipoles $C_{l}$ of the cosmic microwave background anisotropy. We start with the gravitational wave background and then apply the theory to primordial density perturbations. We derive an analytical formula for the positions of peaks and dips in the angular power spectrum $l(l+1) C_{l}$ as a function of $l$. This formula shows that the values of $l$ at the peak positions are ordered in the proportion $1: 3: 5: \ldots$, whereas at the dips they are ordered as $1: 2: 3: \ldots$. We compare the derived positions with the actually observed features, and find them to be in reasonably good agreement. It appears that the observed structure is better described by our analytical formula based on the (squeezed) metric perturbations associated with the primordial density perturbations, rather than by the acoustic peaks reflecting the existence of plasma sound waves at the last scattering surface. We formulate a forecast for other features in the angular power spectrum that may be detected by the advanced observational missions, such as the Microwave Anisotropy Probe and Planck. We tentatively conclude that the observed structure is a macroscopic manifestation of squeezing in the primordial metric perturbations.
\end{abstract}

DOI: 10.1103/PhysRevD.66.043529

PACS number(s): 98.80.Cq, 04.30.-w, 42.50.Dv, 98.70.Vc

\section{INTRODUCTION}

A direct search for relic gravitational waves is one of the important goals of the forthcoming gravitational wave observations (for reviews, see Refs. [1-3]). Relic gravitational waves are inevitably generated by a strong variable gravitational field of the very early Universe through the mechanism of superadiabatic (parametric) amplification of the zero-point quantum oscillations [4]. The word "superadiabatic" emphasizes the fact that this effect takes place over and above whatever effects occur during very slow (adiabatic) changes. That is, we are interested in the increase of occupation numbers, rather than in the gradual shift of energy levels. The word "parametric" emphasizes the underlying mathematical structure of the wave equations. It is a sufficiently quick change of a parameter of the oscillator, namely, variation of its properly defined frequency, that is responsible for the considerable increase of energy of that oscillator.

Apparently, Schrödinger [5] was the first to notice the "alarming phenomenon" in an expanding universe. Specifi-

\footnotetext{
*Permanent address: Department of Physics, Washington State University, 1245 Webster, Pullman, WA 99164-2814; electronic address: sukanta@wsu.edu

†Electronic address: grishchuk@astro.cf.ac.uk
}

cally, Schrödinger discusses the "mutual adulteration of positive and negative frequency terms in the course of time." The frequency mixing means that a traveling wave can be amplified, with the simultaneous appearance of a "reflected" wave, i.e., a wave traveling in the opposite direction. After Schrödinger, out of unawareness of his work, this effect has been rediscovered several times. Schrödinger speaks about the mutual adulteration of electromagnetic waves, which would mean the generation of photons. We now know that the coupling of the electromagnetic field to gravity is such that the generation of photons is impossible, so that the alarming phenomenon does not take place. A detailed study of the Schrödinger paper shows that, in fact, he was operating with a variant of scalar electrodynamics, that is, with a scalar wave equation in an expanding universe model (for a discussion of this point, see Ref. [6]). Then, indeed, the coupling of a scalar field to gravity can be chosen in such a way (minimal coupling), that the generation of scalar particles becomes possible. Parker [7] undertook a systematic study of the quantized version of the scalar wave equation in FLRW (Friedmann-Lemaitre-Robertson-Walker) cosmologies. For a summary of the subject, see Refs. [8,9]. As for gravitational waves, there is no ambiguity in their coupling to gravity because the coupling follows directly from the Einstein equations. It was shown that the gravitational wave equation for each of the two polarization components is exactly the same as the equation for the minimally coupled massless 
scalar field [4]. The early studies were concerned with free test fields superimposed on a given space-time, whereas we are interested in fields arising in the context of perturbed Einstein equations (cosmological perturbations). This distinction is especially important for the issue of quantum normalization of the fields.

Already at this elementary level of discussion, one can make an important observation that will play a crucial role in our study below. If a classical traveling wave, of any physical nature, is going to be strongly amplified, the resulting wave field will form an almost standing wave. A traveling wave can never convert itself into a strict standing wave because of the conservation of linear momentum. But the final amplitudes of the amplified left-moving and rightmoving waves will be large and almost equal, so they interfere to form a practically standing wave.

The amplification process is linear, and the final amplitude of a classical wave is proportional to the initial amplitude. If the amplitude of a classical oscillator is zero initially, the oscillator will not get excited by the parametric influence. However, a quantum oscillator in its vacuum state does possess tiny "zero-point" quantum oscillations. One can think of these vacuum oscillations as the ones that are being amplified. The generation of relic gravitational waves (as well as the generation of other cosmological perturbations, discussed below) is a genuine quantum-gravity process, in the sense that the final result inherently contains all the fundamental constants $\hbar, G$, and $c$. The gravitational energymomentum tensor contains $G$ and $c$, while the Planck constant $\hbar$ enters through the requirement of having initial energy $\frac{1}{2} \hbar \omega$ per mode of the perturbation field. The fundamental constants naturally combine in the Planck length $l_{P l}$ $=\left(G \hbar / c^{3}\right)^{1 / 2}$ or the Planck mass $m_{P l}=(\hbar c / G)^{1 / 2}$, but $l_{P l}$ or $m_{P l}$ must stay in the numerator of the final expression, not in its denominator, so that the final result vanishes if $\hbar$ is formally sent to zero.

The gravitational field of a FLRW universe is given by the metric

$$
\begin{aligned}
\mathrm{d} s^{2} & =-c^{2} \mathrm{~d} t^{2}+a^{2}(t) g_{i j} \mathrm{~d} x^{i} \mathrm{~d} x^{j} \\
& =a^{2}(\eta)\left[-\mathrm{d} \eta^{2}+g_{i j} \mathrm{~d} x^{i} \mathrm{~d} x^{j}\right],
\end{aligned}
$$

where the scale factor $a(t)$ [or $a(\eta)]$ is driven by matter distribution with some effective (in general, time-dependent) equation of state. The scale factor has the dimensionality of length, while $\eta$ and $x^{i}$ are dimensionless. Without restricting in any way the physical content of the problem, one can write the perturbed gravitational field of a FLRW universe (for simplicity, spatially flat) as

$$
\begin{aligned}
\mathrm{d} s^{2}= & a^{2}(\eta)\left[-\mathrm{d} \eta^{2}+\left(\delta_{i j}+h_{i j}\right) \mathrm{d} x^{i} \mathrm{~d} x^{j}\right], \\
h_{i j}(\eta, \mathbf{x})= & \frac{\mathcal{C}}{(2 \pi)^{3 / 2}} \int_{-\infty}^{\infty} d^{3} \mathbf{n} \sum_{s=1,2} \stackrel{s}{p_{i j}}(\mathbf{n}) \frac{1}{\sqrt{2 n}} \\
& \times\left[\stackrel{s}{h}_{n}(\eta) e^{i \mathbf{n} \cdot \mathbf{x}^{s}}{ }_{\mathbf{n}_{\mathbf{n}}}+\stackrel{s}{h}_{n}^{*}(\eta) e^{-i \mathbf{n} \cdot \mathbf{x}} c_{n}^{s_{\dagger}^{\dagger}}\right] .
\end{aligned}
$$

The functions $h_{i j}(\eta, \mathbf{x})$ have been expanded over spatial Fourier harmonics $e^{ \pm i \mathbf{n} \cdot \mathbf{x}}$, where $\mathbf{n}$ is a constant (timeindependent) wave vector. The wave number, $n$, is related to n by $n=\left(\delta_{i j} n^{i} n^{j}\right)^{1 / 2}$. The wave number $n$ defines the wavelength measured in units of laboratory standards (so to say, in centimeters) by $\lambda=2 \pi a / n$. Using the Fourier expansion, we are able to reduce the perturbed dynamical problem to the evolution of mode functions $h_{n}(\eta)$ for each mode $\mathbf{n}$. Two polarization tensors $\stackrel{s}{p}_{i j}(\mathbf{n}), s=1,2$ have different forms depending on whether they represent gravitational waves, rotational perturbations, or density perturbations. If $(\mathbf{n} / n, \mathbf{l}, \mathbf{m})$ are three unit and mutually orthogonal (spatial) vectors, then we have for gravitational waves,

$$
\begin{aligned}
& \stackrel{1_{p}}{p_{i j}}=l_{i} l_{j}-m_{j} m_{i}, \quad \stackrel{2}{p}_{i j}=l_{i} m_{j}+l_{j} m_{i}, \\
& \stackrel{s}{p}_{i j} \delta^{i j}=0, \quad \stackrel{s}{p_{i j}} n^{j}=0,
\end{aligned}
$$

for rotational perturbations,

$$
\begin{aligned}
\stackrel{1}{p}_{i j} & =\frac{1}{n}\left(l_{i} n_{j}+l_{j} n_{i}\right), \quad \stackrel{2}{p}_{i j}=\frac{1}{n}\left(m_{i} n_{j}+m_{j} n_{i}\right), \\
\stackrel{s}{p}_{i j} \delta^{i j} & =0, \quad \stackrel{s}{p}_{i j} n^{i} n^{j}=0,
\end{aligned}
$$

and for density perturbations,

$$
\stackrel{1}{p_{i j}}=\sqrt{\frac{2}{3}} \delta_{i j}, \quad \stackrel{2}{p}_{i j}=-\sqrt{3} \frac{n_{i} n_{j}}{n^{2}}+\frac{1}{\sqrt{3}} \delta_{i j} .
$$

In all three cases, $\stackrel{s}{p}_{i j}(\mathbf{n})$ obey

$$
\stackrel{s}{p}_{i j}(\mathbf{n}) \stackrel{s}{p}^{i j}(\mathbf{n})=2 \delta_{s s^{\prime}}, \quad \stackrel{s}{p}_{i j}(-\mathbf{n})=\stackrel{s}{p}_{i j}(\mathbf{n}) .
$$

In general relativity, rotational and density perturbations can only exist if they are supported by the corresponding perturbations of matter. Their propagation speeds depend on the properties of matter and can range from zero to the speed of light, $c$. For instance, the propagation speed of density perturbations in the radiation-dominated fluid is $c / \sqrt{3}$; and it was very close to $c$ if the very early universe was driven by a scalar field [11]. However, in alternative theories of gravity, solutions with the polarization structure of rotational and density perturbations can exist even in the absence of matter fields, in which case the metric perturbations represent gravitational waves with new polarization states, in addition to the usual gravity-wave polarization states of general relativity [10]. If one concentrates on metric perturbations alone, temporarily leaving aside the accompanying perturbations of matter variables, then all three types of cosmological perturbations in general relativity can be thought of as gravitational waves, even though some of them have unusual polarization states and unusual propagation speeds. There is no wonder that the dynamical equations for cosmological rotational and 
density perturbations are similar to, and sometimes exactly the same as, equations for cosmological gravitational waves. The common "master equation," whose solutions allow one to derive all the metric components along with all the matter perturbations (when they are present), has the universal form [11]:

$$
f^{\prime \prime}+f\left[n^{2} \frac{c_{l}^{2}}{c^{2}}-W(\eta)\right]=0,
$$

where ${ }^{\prime}:=\mathrm{d} / \mathrm{d} \eta, c_{l}$ is a function of $\eta$ and is interpreted as the propagation speed of the perturbation, and $W(\eta)$ is a function of $a(\eta)$ and its derivatives. For density perturbations in a perfect fluid with the fixed equation of state $p=w \epsilon, c_{l}$ is a constant. In the case of gravitational waves, $c_{l}^{2} / c^{2}=1$ and $W(\eta)=a^{\prime \prime} / a$ [4]. One can view Eq. (1.4) as the equation of an oscillator with variable frequency (the term in square brackets), or as the Schrödinger equation of a particle moving in the presence of a potential barrier $W(\eta)$ (while remembering that $\eta$ is a time coordinate rather than a spatial coordinate). In what follows, we will be discussing gravitational waves and density perturbations.

For a classical gravitational field, the quantities $\stackrel{s}{c_{\mathbf{n}}},{\stackrel{s}{c_{\mathbf{n}}}}_{\text {in }}$ Eq. (1.3) are arbitrary complex-conjugate numbers. The constant $\mathcal{C}$ can be incorporated into them. In the quantized version, the quantities $\stackrel{s}{c_{\mathbf{n}}}, \stackrel{s}{\dagger}_{\mathbf{n}}^{\dagger}$ are annihilation and creation operators satisfying the conditions

$$
\left[\begin{array}{c}
s^{\prime} \\
c_{\mathbf{n}}, c_{\mathbf{m}}^{\dagger}
\end{array}\right]=\delta_{s^{\prime} s} \delta^{3}(\mathbf{n}-\mathbf{m}), \quad \stackrel{s}{c_{\mathbf{n}}}|0\rangle=0,
$$

where $|0\rangle$ (for each $\mathbf{n}$ and $s$ ) is the fixed initial vacuum state defined at some $\eta_{0}$ in the very distant past, long before the superadiabatic regime for the given mode has started. In that early era, the mode functions $h_{n}(\eta)$ behaved as $\propto e^{-i n \eta}$, so that each mode $\mathbf{n}$ represented a strict traveling wave propagating in the direction of $\mathbf{n}$. The normalization constant $\mathcal{C}$ is $\sqrt{16 \pi} l_{P l}$ for gravitational waves, and $\sqrt{24 \pi} l_{P l}$ for density perturbations.

A detailed study shows [12] that the quantum-mechanical Schrödinger evolution brings the initial vacuum state of cosmological perturbations into the final multiquantum state known as the squeezed vacuum state. It is the variance of phase that is being strongly diminished (squeezed), while the mean number of quanta and its variance are being strongly increased. A squeezed vacuum state is conveniently characterized by the squeeze parameter $r$. The squeeze parameter grows from $r=0$ in the vacuum state up to $r \gg 1$ by the end of the amplifying superadiabatic regime. The mean number of quanta in a 2-mode squeezed vacuum state is $\langle N\rangle$ $=2 \sinh ^{2} r$. Squeezed vacuum states possess specific statistical properties. In particular, the generated field, viewed as a random field, obeys the statistics of a Gaussian nonstationary process. The nonstationarity means that the variance of the field is an explicit oscillatory function of time, and the twotime correlation function depends on individual moments of time, not only on the time difference. The calculation of quantum-mechanical expectation values and correlation functions provides the link between quantum mechanics and macroscopic physics.

Using the representation (1.3) and definitions above, one finds the variance of metric perturbations:

$$
\left\langle 0\left|h_{i j}(\eta, \mathbf{x}) h^{i j}(\eta, \mathbf{x})\right| 0\right\rangle=\frac{\mathcal{C}^{2}}{2 \pi^{2}} \int_{0}^{\infty} n^{2} \sum_{s=1,2}\left|h_{n}(\eta)\right|^{2} \frac{\mathrm{d} n}{n} .
$$

The quantity

$$
h^{2}(n, \eta)=\frac{\mathcal{C}^{2}}{2 \pi^{2}} n^{2} \sum_{s=1,2}\left|h_{n}(\eta)\right|^{2}
$$

gives the mean-square value of the gravitational field perturbations in a logarithmic interval of $n$ and is called the (dimensionless) power spectrum. In the case of gravitational waves, it is relatively easy to evolve the mode functions up to the present era, and to find that

$$
h^{2}(n, \eta) \propto \sin ^{2}\left[n\left(\eta-\eta_{e}\right)\right],
$$

where $\eta_{e}$ is a constant discussed below. The explicit timedependence of the power spectrum is a consequence of squeezing and can be also viewed as a reflection of the standing-wave pattern of the generated field. For every fixed moment of time (for instance, today) the power spectrum contains many maxima and zeros at certain wave numbers, even though the spectrum was perfectly smooth before amplification, i.e., when the mode functions $\stackrel{s}{h}_{n}(\eta)$ behaved as $\propto e^{-i n \eta}$. As soon as the amplifying process takes place, the increase of the mean number of quanta, squeezing, nonstationarity, formation of standing wave pattern and oscillatory features in the power spectrum, are all the different facets of the same phenomenon.

The relative spacing of zeros is very dense at laboratory scales (large $n$ 's), but is quite sparse at cosmological scales (small $n$ 's). Specifically, the spectrum contains about $10^{20}$ zeros in the interval from 100 to $200 \mathrm{~Hz}$, but only a dozen of zeros in the interval from 1000 to $2000 \mathrm{Mpc}$. The oscillatory time-dependence (1.8) is known in advance, and this information would certainly help, in a very narrow-band gravitational wave detector, to find the signal against the instrumental noise, and to provide evidence for the primordial origin of the detected gravitational wave background [3]. However, in a broadband detector, there are too many zeros together, and the nonstationary process is practically indistinguishable from the stationary process of the same power density. In a recent paper, Allen, Flanagan, and Papa [13] agree that the nonstationarity in the relic background is present, but they argue that this feature can hardly give any advantage in practice. Without presently being able to offer a realistic scheme of exploiting the nonstationarity at small scales, we shift our attention to cosmological scales, where the spacing of zeros is sparse. The natural place to look for the consequences of squeezing is the distribution of multipoles $C_{\ell}$ of the cosmic microwave background (CMB) anisotropies [14]. 
Relic gravitational waves should be important at lower multipoles $\ell$ of the CMB anisotropies, but they are not expected to give significant contribution at $\ell \sim 200$ and higher. It is the density perturbations that are expected to be primarily responsible for the behavior of the $C_{\ell}$ 's in the latter range. However, we study in great detail the simpler case of gravitational waves in order to resolve a number of principal issues and to get a guidance for the analysis of the technically more difficult case of density perturbations. The phenomenon of squeezing is universal, and if the primordial density perturbations have a quantum-mechanical origin (which, we believe, is likely to be true), then many features must be common with the case of relic gravitational waves. Some differences arise at the late stages of evolution (in particular, they explain why the gravitational wave (gw) contribution is subdominant at $\ell \sim 200$ and higher), but we take them into account. Developing the conjecture of Ref. [15], we argue that it is the modulated structure of the metric power spectrum (which is caused, on a fundamental level, by squeezing) that is responsible for the downturn of the rising function $\ell(\ell+1) C_{\ell}$ at the peak, and for the appearance of subsequent peaks and dips, a few of which have been recently observed [16]. On the ground of our simple analytical treatment we make a forecast for the positions of further peaks and dips that may be observed by future missions, such as the Microwave Anisotropy Probe (MAP) and Planck. In general, our forecast agrees with that of Ref. [17], made on the grounds of numerical codes, but discrepancies become significant somewhere around the fourth expected peak.

The structure of the paper and its conclusions are as follows. We start, in Sec. II, with the gravitational wave solutions in the present universe, that is, at the matter-dominated stage. We consider the general solution for the time dependent mode functions $h_{n}(\eta)$, regardless of whether a given particular solution is likely to emerge from the very early universe or not. In general, a given mode $\mathbf{n}$ is neither a traveling wave nor a standing wave. We formulate conditions under which a given mode is a strict traveling wave or a strict standing wave. These conditions are constraints on the (Fourier) coefficients $A_{n}, B_{n}$ in front of two linearly independent solutions for the mode functions. Then, we explore the issue of stationary versus nonstationary variance. We demonstrate that oscillations in the power spectrum are most pronounced when the modes are standing waves, and they disappear when the modes are traveling waves. Thus the often made (incorrect) statement that the relic gravitational wave background should be stationary is equivalent to the assumption that it is being formed by traveling waves. We return to this issue later on (in Sec. IV) and show that, whether the nonstationarity on small scales is measurable or not, the very assumption of the stationary gravitational wave background is in conflict with some other cosmological considerations. In Sec. III we present a "physical" model for the gravitational wave background. We call it "physical" because we evolve the field through all three relevant stages of cosmological evolution: initial, radiation-dominated, and matterdominated. In this way we distinguish the "physical" model from the "alternative" model, which postulates that the gravitational wave background is stationary, irrespective of its physical origin. The waves generated in the physical model are squeezed (standing). We demonstrate that the evolution of standing waves through the effective barrier $a^{\prime \prime} / a$ at the matter-dominated stage results in the appearance of an oscillatory behavior of the Fourier coefficients $A_{n}, B_{n}$ as functions of the wave number $n$. We later show (in Sec. V) that this oscillatory behavior of $A_{n}, B_{n}$ is the origin of oscillations in the multipole moment distribution $C_{\ell}$ as a function of $\ell$.

Section IV compares the physical and alternative gravitational wave backgrounds. We introduce the notion of a fair comparison, which requires that today's band-powers of the two backgrounds be equal at all scales. By evolving the corresponding solutions backwards in time, we show that the alternative background would have had too much power in long waves at the era of last scattering of the CMB radiation. In terms of "growing" and "decaying" solutions, this means that the amplitude of the decaying solution in the alternative background becomes dangerously large when one returns deeper and deeper into the past. The further evolution backwards in time would have destroyed our sacred belief that the Universe was homogeneous and isotropic (up to small perturbations) at the time of the primordial nucleosynthesis and its past. Most importantly for our study, we demonstrate that the alternative background does not produce oscillations in the $C_{\ell}$ multipoles. This shows that the squeezing is observationally distinguishable, even if, at the present level of observational capabilities, it is better to search on large scales rather than on small scales.

Section $\mathrm{V}$ presents the results of numerical calculations of the $C_{\ell}$ 's caused by relic gravitational waves. We show that our analytical formula for the positions of peaks and dips is in a fairly good agreement with numerical calculations. This analysis demonstrates that, at least in the case of gravitational waves, the $C_{\ell}$ oscillations are produced by modulations in the power spectrum of metric perturbations, and not by acoustic waves at the last scattering surface, simply because there are no matter perturbations at all.

In Sec. VI, we turn to the primordial density perturbations. The evolution of density perturbations through the initial and radiation-dominated stages is almost identical to the evolution of gravitational waves. We show that the Fourier coefficients $A_{n}, B_{n}$ of metric perturbations associated with density perturbations develop a periodic structure, as functions of $n$, in the course of transition from the radiationdominated phase to the matter-dominated phase. In a manner similar to the gravitational wave case, we derive the expected positions of peaks and dips in the $C_{\ell}$ distribution. Because of the damping, features beyond the second peak may not be easily discernible [18-20], but they seem to be less likely to be washed out if they are produced by modulations in the metric power spectrum rather than by modulations in the plasma matter power spectrum. We show that the peak positions should obey the rule $1: 3: 5: 7 \ldots$, whereas the dip positions should be ordered as $1: 2: 3: 4 \ldots$ We demonstrate that the observed positions agree better with our analytical formula than with the concept of "acoustic peaks." The detection of late features may be especially interesting as our 
analytical forecast is somewhat different from what follows from conventional numerical codes. We tentatively conclude that the observed structures in the angular power spectrum $\ell(\ell+1) C_{\ell}$ are macroscopic manifestations of squeezing in gravitational field perturbations.

\section{PROPERTIES OF GRAVITATIONAL WAVE SOLUTIONS}

The perturbed Einstein equations for gravitational waves can be reduced to the "master equation"

$$
\stackrel{s}{\mu_{n}^{\prime \prime}}+\stackrel{s}{\mu_{n}}\left[n^{2}-\frac{a^{\prime \prime}}{a}\right]=0,
$$

where the functions $\stackrel{s}{\mu_{n}}(\eta)$ are related to the mode functions $\stackrel{s}{h_{n}}(\eta)$ by

$$
\stackrel{s}{\mu}{ }_{n}(\eta) \equiv a(\eta) \stackrel{s}{h}_{n}(\eta) .
$$

We suppress the polarization index $s$ when it causes no ambiguity.

The scale factor $a(\eta)$ at the matter-dominated stage, governed by whatever matter with the effective equation of state $p=0$, behaves as $a(\eta) \propto \eta^{2}$. It is convenient to write $a(\eta)$ in the explicit form

$$
a(\eta)=2 l_{H}\left(\eta-\eta_{m}\right)^{2},
$$

where $l_{H}$ is the Hubble radius today $\left(l_{H}=c / H_{0}\right.$, where $H_{0}$ is the present value of the Hubble parameter) and $\eta_{m}$ is a constant explained below. The moment of time "today" (in the cosmological sense) is labeled by $\eta=\eta_{R}$ (the subscript $R$ denoting "reception"). It is convenient to choose

$$
\eta_{R}-\eta_{m}=1
$$

With this convention, $a\left(\eta_{R}\right)=2 l_{H}$, and the wave, of any physical nature, whose wavelength $\lambda$ today is equal to today's Hubble radius, carries the constant wave number $n_{H}$ $=4 \pi$. Longer waves have smaller $n$ 's and shorter waves have larger $n$ 's, according to the relationship $n=4 \pi l_{H} / \lambda$. For example, the ground-based gravitational wave detectors are most sensitive to frequencies around $30-3000 \mathrm{~Hz}$. The corresponding wavelengths have wave numbers $n$ somewhere in the interval $10^{20}-10^{22}$.

For the scale factor (2.3), Eq. (2.1) is easily solved to yield

$$
\begin{aligned}
\mu_{n} & =\sqrt{y}\left[A_{n} J_{3 / 2}(y)-i B_{n} J_{-3 / 2}(y)\right] \\
& \equiv \frac{\sqrt{y}}{2}\left[\left(A_{n}-B_{n}\right) H_{3 / 2}^{(1)}(y)+\left(A_{n}+B_{n}\right) H_{3 / 2}^{(2)}(y)\right],
\end{aligned}
$$

where

$$
y \equiv n\left(\eta-\eta_{m}\right)
$$

and $J_{ \pm 3 / 2}(y)$ and $H_{3 / 2}^{(1,2)}(y)$ are Bessel and Hankel functions, respectively [21]. We will also be using spherical Bessel functions $j_{i}(y)=\sqrt{\pi / 2 y} J_{i+1 / 2}(y)$. The (Fourier) coefficients $A_{n}$ and $B_{n}$ are, so far, arbitrary complex numbers. In the limit $y \rightarrow \infty$,

$$
\begin{aligned}
& H_{3 / 2}^{(1)}(y) \sim-\sqrt{\frac{2}{\pi y}} e^{i y}, \quad H_{3 / 2}^{(2)}(y) \sim-\sqrt{\frac{2}{\pi y}} e^{-i y} \\
& \text { and } j_{1}(y) \sim-\frac{1}{y} \cos y, \quad j_{-2}(y) \sim-\frac{1}{y} \sin y .
\end{aligned}
$$

Thus, at late times, $\mu_{n}(\eta)$ is a combination of sine and cosine functions of time:

$$
\begin{aligned}
\mu_{n}(\eta) & =-\frac{1}{\sqrt{2 \pi}}\left[\left(A_{n}-B_{n}\right) e^{i y}+\left(A_{n}+B_{n}\right) e^{-i y}\right] \\
& =-\sqrt{\frac{2}{\pi}}\left(A_{n} \cos y-i B_{n} \sin y\right)
\end{aligned}
$$

\section{A. Standing versus traveling waves}

We now consider a classical field (1.3). The $\mathbf{n}$ th mode of the field is given by the real function

$$
h_{\mathbf{n}}(\eta, \mathbf{x}) \equiv h_{n}(\eta) e^{i \mathbf{n} \cdot \mathbf{x}} c_{\mathbf{n}}+h_{n}^{*}(\eta) e^{-i \mathbf{n} \cdot \mathbf{x}} c_{\mathbf{n}}^{*},
$$

where a complex number $c_{\mathbf{n}}$ can be conveniently presented in its polar form:

$$
c_{\mathbf{n}} \equiv \rho_{c_{\mathbf{n}}} e^{i \phi_{c_{\mathbf{n}}}}
$$

The gravitational wave solutions at late times are given by Eq. (2.8). Thus we have

$$
\begin{aligned}
h_{\mathbf{n}}(\eta, \mathbf{x}) \approx & -\frac{\rho_{c_{\mathbf{n}}}}{a(\eta)} \sqrt{\frac{2}{\pi}}\left[\left(A_{n} \cos y-i B_{n} \sin y\right)\right. \\
& \left.\times e^{i\left(\mathbf{n} \cdot \mathbf{x}+\phi_{c_{\mathbf{n}}}\right)}+\left(A_{n}^{*} \cos y+i B_{n}^{*} \sin y\right) e^{-i\left(\mathbf{n} \cdot \mathbf{x}+\phi_{c_{\mathbf{n}}}\right)}\right] .
\end{aligned}
$$

If the coefficients $A_{n}, B_{n}$ are arbitrary, Eq. (2.11) is neither a traveling wave nor a standing wave. A traveling wave is characterized by two real numbers, namely, an amplitude $A$ and a phase $\phi$; its general form is $A \sin ( \pm n \eta+\mathbf{n} \cdot \mathbf{x}+\phi)$. The minus/plus sign describes a wave traveling in the positive/ negative direction defined by the fixed vector $\mathbf{n}$. A standing wave is characterized by three real numbers, viz., an amplitude $A$ and two phases, and its general form is $A \sin (n \eta$ $\left.+\phi_{1}\right) \sin \left(\mathbf{n} \cdot \mathbf{x}+\phi_{2}\right)$. Different choices of these free parameters are responsible for concrete space-time patterns, but they do not change the wave classification.

A little investigation shows that Eq. (2.11) describes a traveling wave if and only if

$$
A_{n}= \pm B_{n}
$$


This constraint can also be written as

$$
A_{n}=\rho_{n} e^{i \phi_{n}} \quad \text { and } \quad B_{n}= \pm \rho_{n} e^{i \phi_{n}}
$$

where $\rho_{n}$ and $\phi_{n}$ are two arbitrary real numbers. We will refer to this constraint as the traveling wave condition. On the other hand, Eq. (2.11) describes a standing wave if and only if

$$
A_{n}=\rho_{A_{n}} e^{i \phi_{n}} \quad \text { and } \quad B_{n}= \pm i \rho_{B_{n}} e^{i \phi_{n}}
$$

where $\rho_{A_{n}}, \rho_{B_{n}}$, and $\phi_{n}$ are three arbitrary real numbers. We will refer to this constraint as the standing wave condition. Two special cases of the standing wave condition are when either $A_{n}=0$ or $B_{n}=0$. This classification of traveling and standing waves was based on the regime when a given mode satisfies the requirement $y \gg 1$ (short-wave regime), but it can now be applied at earlier times too, when this requirement is not satisfied (long-wave regime).

\section{B. Stationary versus nonstationary variance}

The variance of a quantized field (1.3) is defined by Eq. (1.6). The essential part of this expression is the power spectrum given by Eq. (1.7). At the matter-dominated stage, the general solution for the gravitational wave mode functions is represented by Eq. (2.5). One can now find the power spectrum. We use spherical Bessel functions and replace the summation over $s$ by the multiplication factor 2 . Then, the general expression for the power spectrum is

$$
\begin{aligned}
h^{2}(n, \eta)= & \frac{2 \mathcal{C}^{2} n^{2} y^{2}}{\pi^{3} a^{2}(\eta)}\left[\left|A_{n}\right|^{2} j_{1}^{2}(y)+\left|B_{n}\right|^{2} j_{-2}^{2}(y)\right. \\
& \left.+2 \operatorname{Im}\left(A_{n}^{*} B_{n}\right) j_{1}(y) j_{-2}(y)\right] .
\end{aligned}
$$

Using the constraints (2.13) and (2.14), one can now specialize the power spectrum to traveling and standing wave cases. In the traveling wave case one obtains

$$
h^{2}(n, \eta)=\frac{2 \mathcal{C}^{2} n^{2} y^{2} \rho_{n}^{2}}{\pi^{3} a^{2}(\eta)}\left[j_{1}^{2}(y)+j_{-2}^{2}(y)\right],
$$

and in the standing wave case,

$$
h^{2}(n, \eta)=\frac{2 \mathcal{C}^{2} n^{2} y^{2}}{\pi^{3} a^{2}(\eta)}\left[\rho_{A_{n}} j_{1}(y) \pm \rho_{B_{n}} j_{-2}(y)\right]^{2} .
$$

When considering the waves that are shorter than the Hubble radius, $y \gg 1$, one can use asymptotic formulas (2.7) for the Bessel functions. One can also replace $a(\eta)$ with $2 l_{H}$, since the scale factor does not practically change during any reasonable observation time. Then, in the traveling wave case, the oscillations of the power spectrum fully disappear, as the oscillating terms $\cos ^{2} y$ and $\sin ^{2} y$ combine to 1 . However, the oscillations are most pronounced in the standing wave case, as Eq. (2.17) exhibits a periodic structure

$$
h^{2}(n, \eta)=\frac{\mathcal{C}^{2} n^{2}}{2 \pi^{3} l_{H}^{2}}\left[\rho_{A_{n}} \cos y \pm \rho_{B_{n}} \sin y\right]^{2}, \quad y \gg 1
$$

In other words, the power spectrum of traveling waves is stationary, whereas the power spectrum of standing waves is nonstationary. These two classes of power spectra are subcases of the general situation in which $A_{n}=\rho_{A_{n}} e^{i \phi_{A_{n}}, B_{n}}$

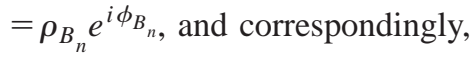

$$
\begin{aligned}
h^{2}(n, \eta)= & \frac{\mathcal{C}^{2} n^{2}}{2 \pi^{3} l_{H}^{2}}\left[\rho_{A_{n}}^{2} \cos ^{2} y+\rho_{B_{n}}^{2} \sin ^{2} y\right. \\
& \left.+2 \rho_{A_{n}} \rho_{B_{n}} \sin \left(\phi_{B_{n}}-\phi_{A_{n}}\right) \sin y \cos y\right],
\end{aligned}
$$

$$
y \gg 1
$$

At a fixed moment of time, for instance today, the power spectrum (2.18) reduces to

$$
h^{2}\left(n, \eta_{R}\right)=\frac{\mathcal{C}^{2} n^{2}}{2 \pi^{3} l_{H}^{2}}\left[\rho_{A_{n}} \cos n \pm \rho_{B_{n}} \sin n\right]^{2}, \quad n \gg 1 .
$$

As one can see, the power spectrum of standing waves contains, quite generically, many maxima and zeros at certain $n$ 's due to the oscillatory factors $\cos n, \sin n$. The coefficients $\rho_{A_{n}}, \rho_{B_{n}}$ are still arbitrary and could, in principle, be smooth functions of $n$. However, as we will show in the next section, the preceding evolution of standing waves through the transition from the radiation-dominated era to the matterdominated era, gives rise to additional oscillations, namely to oscillations in the coefficients $\rho_{A_{n}}, \rho_{B_{n}}$ themselves, as functions of $n$.

\section{THE PHYSICAL MODEL FOR RELIC GRAVITATIONAL WAVE BACKGROUND}

\section{A. The behavior of the scale factor: Pump field}

The matter-dominated $(m)$ era with the scale factor $(2.3)$ was preceded by the radiation-dominated $(e)$ era with the scale factor $a(\eta) \propto \eta$. To simplify the analysis, and without any essential loss of generality, we assume that the transition from $e$ era to $m$ era was instantaneous and took place at some $\eta=\eta_{2}$. The redshift of the transition is $z_{e q}: a\left(\eta_{R}\right) / a\left(\eta_{2}\right)$ $=1+z_{e q}$. It is believed that $z_{e q}$ is somewhere near $6 \times 10^{3}$. In its turn, the radiation-dominated era was preceded by the initial $(i)$ era of expansion, whose nature and scale factor are, strictly speaking, unknown. To simplify the analysis, and since the wave equations admit simple exact solutions in case of power-law scale factors, we assume that the $i$ era, similar to the $e$ and $m$ eras, was also described by a powerlaw scale factor. We parametrize the $i$ era by $a(\eta) \propto|\eta|^{1+\beta}$ (compare with [4]). The transition from $i$ era to $e$ era takes place at some $\eta=\eta_{1}$ and at redshift $z_{i}: a\left(\eta_{R}\right) / a\left(\eta_{1}\right)=1$ $+z_{i}$. Further analysis shows (see below) that in order to get 
the right amplitude of the generated perturbations, the numerical value of $z_{i}$ should be somewhere near $10^{30}$.

We now write the full evolution of the growing scale factor explicitly:

$$
\begin{aligned}
& a(\eta)=l_{o}|\eta|^{1+\beta}, \quad \eta \leqslant \eta_{1}, \quad \eta_{1}<0, \quad \beta<-1, \\
& a(\eta)=l_{o} a_{e}\left(\eta-\eta_{e}\right), \quad \eta_{1} \leqslant \eta \leqslant \eta_{2}, \\
& a(\eta)=2 l_{H}\left(\eta-\eta_{m}\right)^{2}, \quad \eta_{2} \leqslant \eta .
\end{aligned}
$$

The continuous joining of $a(\eta)$ and $a^{\prime}(\eta)$ at the transition points fully determines all the participating constants in terms of $l_{H}, z_{i}, z_{e q}$, and $\beta$. Concretely,

$$
\begin{aligned}
& \eta_{R}=1+\eta_{m}, \quad \eta_{m}=-\frac{1}{2 \sqrt{1+z_{e q}}}\left[1-\beta \frac{1+z_{e q}}{1+z_{i}}\right], \\
& \eta_{2}=\frac{1}{2 \sqrt{1+z_{e q}}}\left[1+\beta \frac{1+z_{e q}}{1+z_{i}}\right], \\
& \eta_{e}=\frac{1}{2} \beta \frac{\sqrt{1+z_{e q}}}{1+z_{i}}, \quad \eta_{1}=\frac{1}{2}(1+\beta) \frac{\sqrt{1+z_{e q}}}{1+z_{i}},
\end{aligned}
$$

and

$$
\begin{aligned}
l_{o} a_{e} & =\frac{4 l_{H}}{\sqrt{1+z_{e q}}}, \\
l_{o} & =l_{H} \frac{2^{2+\beta}}{|1+\beta|^{1+\beta}} \frac{\left(1+z_{e q}\right)^{-(\beta+1) / 2}}{\left(1+z_{i}\right)^{-\beta}} .
\end{aligned}
$$

The case $\beta=-2$ is known as the de Sitter inflation. In this particular case,

$$
\left.l_{o}\right|_{\beta=-2}=l_{H} \frac{\sqrt{1+z_{e q}}}{\left(1+z_{i}\right)^{2}} .
$$

For the CMB calculations we will also need the redshift $z_{\text {dec }}$ of the last scattering surface $\eta=\eta_{E}$ (with the subscript $E$ denoting "emission"), where the CMB photons have decoupled from rest of the matter: $a\left(\eta_{R}\right) / a\left(\eta_{E}\right)=1+z_{d e c}$. The numerical value of $z_{d e c}$ is somewhere near 1000 . The time of decoupling $\eta_{E}$ is

$$
\eta_{E}=\frac{1}{\sqrt{1+z_{d e c}}}-\frac{1}{2 \sqrt{1+z_{e q}}}+\beta \frac{\sqrt{1+z_{e q}}}{2\left(1+z_{i}\right)} .
$$

All the formulas above are exact and we will be using them often, but surely one can get an excellent approximation by neglecting 1 in comparison with $z_{i}, z_{e q}$, and $z_{d e c}$.

\section{B. Squeezed gravitational waves}

As soon as the scale factor and initial conditions for the mode functions are strictly defined, the coefficients $A_{n}, B_{n}$ in Eq. (2.5) are strictly calculable [22,23]. The general solution for $\mu_{n}$ at the $e$ stage is

$$
\mu_{n}(\eta)=B_{1} e^{-i n\left(\eta-\eta_{e}\right)}+B_{2} e^{i n\left(\eta-\eta_{e}\right)},
$$

but the preceding evolution allows one to specify $B_{1}$ and $B_{2}$. The waves subject to amplification at the $i$ stage have the wave numbers $n$ satisfying the condition $n\left|\eta_{1}\right| \ll 2 \pi|1+\beta|$, that is,

$$
\frac{n}{n_{H}} \ll \frac{1+z_{i}}{\sqrt{1+z_{e q}}} .
$$

For these waves, one finds

$$
B_{1} \approx-B_{2} \approx F(\beta)\left(\frac{n \sqrt{1+z_{e q}}}{1+z_{i}}\right)^{\beta} \equiv B,
$$

where

$$
F(\beta)=-e^{i\left(x_{0}+\pi \beta / 2\right)} \frac{\sqrt{\pi}|1+\beta|^{\beta+1}}{2^{2(\beta+1)} \Gamma(\beta+3 / 2) \cos \beta \pi},
$$

and $x_{0} \equiv n \eta_{0}[22]$. Note that in the particular case $\beta=-2$,

$$
|B|^{2}=\frac{4\left(1+z_{i}\right)^{4}}{n^{4}\left(1+z_{e q}\right)^{2}}, \quad \beta=-2 .
$$

The fact that $B_{1} \approx-B_{2}$ demonstrates that the gravitational wave modes $\mathbf{n}$ are (almost) standing waves at the $e$ stage. These standing waves are encountering the nonzero barrier $a^{\prime \prime} / a$ at the $m$ stage. To find the coefficients $A_{n}, B_{n}$ in Eq. (2.5) one needs to join continuously the $\mu_{n}(\eta)$ and $\mu_{n}^{\prime}(\eta)$ at the transition point $\eta=\eta_{2}$. This calculation allows one to find the coefficients $A_{n}, B_{n}$, but it also shows (as expected) that the standing-wave character of the field at the $e$ stage leads to the appearance of oscillations in the coefficients $A_{n}$, $B_{n}$ of the field at the $m$ stage. This is a general phenomenon which we will also discuss in connection with density perturbations. Explicitly,

$$
\begin{aligned}
& A_{n}=-i \sqrt{\frac{\pi}{2} \frac{B}{4 y_{2}^{2}}\left[\left(8 y_{2}^{2}-1\right) \sin y_{2}+4 y_{2} \cos y_{2}+\sin 3 y_{2}\right]} \\
& B_{n}=-\sqrt{\frac{\pi}{2}} \frac{B}{4 y_{2}^{2}}\left[\left(8 y_{2}^{2}-1\right) \cos y_{2}-4 y_{2} \sin y_{2}+\cos 3 y_{2}\right]
\end{aligned}
$$

where

$$
y_{2}=n\left(\eta_{2}-\eta_{e}\right)=\frac{n}{n_{m}}, \quad \text { and } \quad n_{m}=2 \sqrt{1+z_{e q}} .
$$

The numerical value of $n_{m}$ is about 160. This corresponds to wavelengths that are 15 times shorter than $l_{H}$. Clearly, the coefficients (3.12) satisfy the standing wave condition (2.14). It is easy to check that had one artificially chosen traveling waves at the $e$ stage, by assuming that either $B_{1}=0$ or $B_{2}$ $=0$, the oscillations in $A_{n}, B_{n}$ would have been suppressed. 
The asymptotic expressions for $A_{n}, B_{n}$ are as follows. For relatively short waves, i.e., $y_{2} \gg 1, n \gg n_{m}$, one has

$$
A_{n} \approx-i 2 \sqrt{\pi / 2} B \sin y_{2}, \quad B_{n} \approx-2 \sqrt{\pi / 2} B \cos y_{2} .
$$

Using these coefficients in Eq. (2.8), one finds the $\mu_{n}(\eta)$ at the $m$ stage, for $n \gg n_{m}$ :

$$
\mu_{n}(\eta) \approx-i 2 B \sin \left[n\left(\eta-\eta_{e}\right)\right]
$$

Of course, this function is simply a continuation of the relevant standing wave solution $\left(B_{1} \approx-B_{2} \approx B\right)$ at the $e$ stage, Eq. (3.8), to the $m$ stage. Indeed, the height of the barrier at the $m$ stage is

$$
\left.\frac{a^{\prime \prime}}{a}\right|_{\eta=\eta_{2}}=\frac{1}{2} n_{m}^{2}
$$

so the waves with $n \gg n_{m}$ stay above the barrier and experience no changes. However, the waves with $n \approx n_{m}$ and $n$ $\ll n_{m}$ are affected by the barrier. For relatively long waves, i.e., $n \ll n_{m}$, one has

$$
A_{n} \approx-i \frac{3 \sqrt{\pi}}{2 \sqrt{2}} B y_{2}^{-1}, \quad B_{n} \approx \frac{8 \sqrt{\pi}}{45 \sqrt{2}} B y_{2}^{4},
$$

such that $\left|B_{n}\right| \ll\left|A_{n}\right|$. The formulas above are in full agreement with [22] if one takes into account the change of notations: $A_{n}=\sqrt{\pi / 2} C_{1 n}, B_{n}=i \sqrt{\pi / 2} C_{2 n}$. In particular, the long-wavelength part of the power spectrum at the time of decoupling $\eta=\eta_{E}$ is given by

$$
h^{2}\left(n, \eta_{E}\right) \approx \frac{4}{\pi} \frac{l_{P l}^{2}}{l_{H}^{2}}\left(1+z_{e q}\right) n^{4}|B|^{2}, \quad n \ll n_{d e c}=\sqrt{1+z_{d e c}} .
$$

This part of the spectrum is primordial, in the sense that it has not changed since the beginning of amplification. In the particular case $\beta=-2$, and using Eq. (3.11), we obtain the flat (independent of $n$ ) primordial spectrum

$$
\begin{aligned}
h^{2}\left(n, \eta_{E}\right) & \approx \frac{16}{\pi} \frac{l_{P l}^{2}}{l_{H}^{2}} \frac{\left(1+z_{i}\right)^{4}}{\left(1+z_{e q}\right)}, \quad \beta=-2, \\
n \ll n_{d e c} & =\sqrt{1+z_{d e c}} .
\end{aligned}
$$

In preparation for the discussion of CMBR anisotropies, we show in Fig. 1 the numerically calculated spectrum $h^{2}\left(n, \eta_{E}\right)$, including the beginning of its oscillations. We use the notations

$$
x \equiv y_{2}=\frac{n}{n_{m}}, \quad b \equiv \frac{n_{m}}{n_{d e c}}=\frac{2 \sqrt{1+z_{e q}}}{\sqrt{1+z_{d e c}}} .
$$

The substitution of $A_{n}, B_{n}$ given by Eq. (3.12) into the general expression (2.15) produces the exact power spectrum

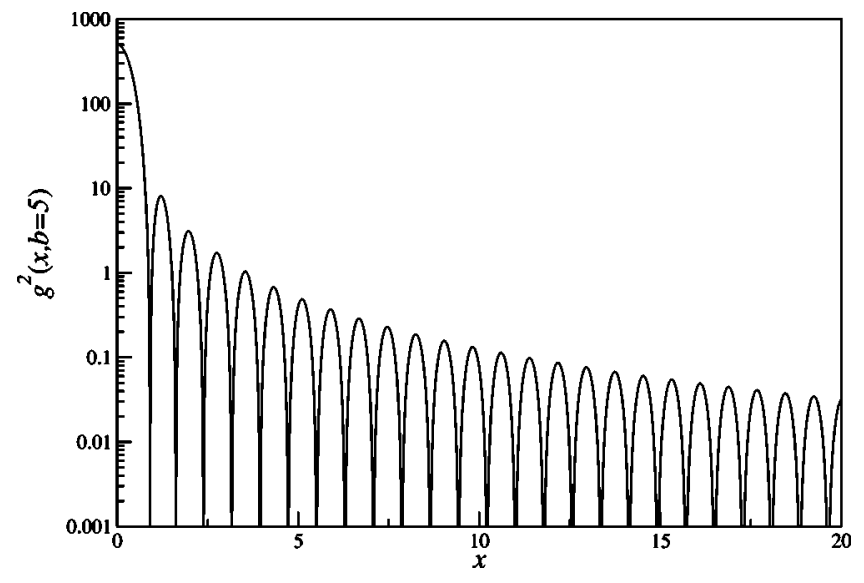

FIG. 1. Plot of $g^{2}(x, b=5)$ vs $x$.

$$
h^{2}\left(n, \eta_{E}\right)=\frac{l_{P l}^{2}}{4 \pi l_{H}^{2}}\left(1+z_{d e c}\right) n^{4}|B|^{2} g^{2}(x, b),
$$

where

$$
\begin{aligned}
g^{2}(x, b) & =\left[\rho_{1}(x) j_{1}(b x)-\rho_{2}(x) j_{-2}(b x)\right]^{2}, \\
x & \equiv \frac{n}{n_{m}}, \quad b \equiv \frac{n_{m}}{n_{d e c}}, \\
\rho_{1}(x) & =\frac{1}{x^{2}}\left[\left(8 x^{2}-1\right) \sin x+4 x \cos x+\sin 3 x\right], \\
\rho_{2}(x) & =\frac{1}{x^{2}}\left[\left(8 x^{2}-1\right) \cos x-4 x \sin x+\cos 3 x\right] .
\end{aligned}
$$

Note that the rigorously evolved mode functions single out the lower sign in the general formula (2.17) for standing waves. Figure 1 shows the function $g^{2}(x, b)$ for $b=5$.

The positions of maxima and zeros of the power spectrum are well approximated by the short-wavelength limit of $g^{2}(x, b)$ :

$$
g^{2}(x, b) \approx \frac{64}{b^{2} x^{2}} \sin ^{2}[(b-1) x] .
$$

In the case of $b=5$, the maxima and zeros of the function $\sin ^{2} 4 x$ are ordered as follows:

$$
\begin{aligned}
\text { Maxima: } \quad x_{k}^{\text {max }} & =\frac{\pi}{8}(2 k+1), \\
x_{k}^{\text {max }} & =x_{0}^{\text {max }}(2 k+1), \quad k=0,1,2,3, \ldots, \\
\text { Zeros: } \quad x_{k}^{\text {min }} & =\frac{\pi}{8} 2 k, \\
x_{k}^{\text {min }} & =x_{1}^{\text {min }} k, \quad k=1,2,3, \ldots .
\end{aligned}
$$

The analytical forecast (3.18),(3.19) shifts the first few features to the left ( $\operatorname{smaller} x)$ as compared with the more ac- 
curate numerical calculation, but the forecast becomes progressively more accurate for later features. For example, the analytical formula predicts $x_{1}^{\min }=0.79, x_{1}^{\max }=1.18, x_{8}^{\min }$ $=6.28, x_{8}^{\max }=6.68$, whereas the numerical calculation places these features at $x_{1}^{\min }=0.91, x_{1}^{\max }=1.21, x_{8}^{\min }=6.29, x_{8}^{\max }$ $=6.68$. The zeroth maximum $x_{0}^{\max }$ would be placed, according to the analytical formula (3.18), at $x_{0}^{\max }=0.393$. The crucial rule, however, is that the positions of minima, starting from $x_{1}^{\min }$, are ordered in the proportion $1: 2: 3: 4 \ldots$, whereas the positions of maxima, starting from the zeroth "would-be" maximum, are ordered in proportion $1: 3: 5: 7 \ldots$. We will see in Sec. $V$ how these features are being reflected in the oscillations of the $C_{l}$ multipoles.

\section{DISTINGUISHING BETWEEN THE PRESENCE AND THE ABSENCE OF SQUEEZING}

As demonstrated above, the quantum-mechanically generated (squeezed) gravitational waves form a nonstationary background, whose power spectrum is fully determined by fundamental constants and parameters of the gravitational pump field. In this section we show that the hypothesis whereby the gravitational wave background is postulated to be stationary, and therefore nonsqueezed, is in conflict with some other cosmological data. Since we concentrate on stationarity versus nonstationarity, the comparison of the two backgrounds should be fair, in the sense that their today's (for $\eta=\eta_{R}$ ) band-powers should be assumed equal. However, as we will show below, when one returns back in time to, say, the decoupling era, the alternative background is bound to have too much power in long-wavelength perturbations. The further extrapolation back in time destroys the usual (and partially tested) assumption that the cosmological perturbations remain small all the way down to the nucleosynthesis era and beyond. In Sec. V we will also show that the alternative background does not produce oscillations in the $C_{l}$ multipoles, whereas the physical background does.

The power spectrum of the physical background is given by the general expression (2.15) where $A_{n}, B_{n}$ are determined by Eq. (3.12). The substitution of Eq. (3.12) in Eq. (2.15) results in formula (2.17) (with the lower sign) where $\rho_{A_{n}}, \rho_{B_{n}}$ are absolute values of the coefficients (3.12). On the other hand, the general form of the power spectrum for the alternative (stationary) background is given by Eq. (2.16), where $\rho_{n}$ should be found from the fair comparison:

$$
\rho_{n}^{2}\left[j_{1}^{2}(n)+j_{-2}^{2}(n)\right]=\left[\rho_{A_{n}} j_{1}(n)-\rho_{B_{n}} j_{-2}(n)\right]^{2} .
$$

We will find $\rho_{n}$ using asymptotic expressions (3.13) and (3.14) in different intervals of $n$. In the region $n \gg n_{m}$, formula (3.13) yields

$$
\rho_{A_{n}} \approx \sqrt{2 \pi}|B|\left|\sin \frac{n}{n_{m}}\right|, \quad \rho_{B_{n}} \approx \sqrt{2 \pi}|B|\left|\cos \frac{n}{n_{m}}\right| .
$$

In the region $n \ll n_{m}$ formula (3.14) yields to

$$
\rho_{A_{n}} \approx \frac{3 \sqrt{\pi}}{2 \sqrt{2}}|B| \frac{n_{m}}{n}, \quad \rho_{B_{n}} \approx \frac{8 \sqrt{\pi}}{45 \sqrt{2}}|B|\left(\frac{n}{n_{m}}\right)^{4} .
$$

Let us start from $n \gg n_{m}$. Using asymptotic expressions (2.7) for the spherical Bessel functions, and replacing $\sin ^{2} n$ with $1 / 2$ in the right-hand side of Eq. (4.1), one finds

$$
\rho_{n}^{2} \approx \pi|B|^{2}, \quad n \gg n_{m} .
$$

Now turn to the interval $n_{m} \gg n \gg 1$. The $\rho_{A_{n}}, \rho_{B_{n}}$ are now given by Eq. (4.3). One can still use the asymptotic formulas (2.7), but the second term in the right-hand side of Eq. (4.1) is much smaller than the first one and therefore can be neglected. Then, one derives

$$
\rho_{n}^{2} \approx \frac{9 \pi}{16}|B|^{2} \frac{n_{m}^{2}}{n^{2}}, \quad n_{m} \gg n \gg 1 .
$$

Finally, in the region $n \ll 1$, one uses asymptotic formulas $j_{1}(n) \sim n / 3, j_{-2}(n) \sim 1 / n^{2}$. In either side of Eq. (4.1), the first term is smaller than the second term, and can be neglected. Then, one obtains

$$
\rho_{n}^{2} \approx \frac{\pi}{8}|B|^{2} n_{m}^{2} n^{4}, \quad n \ll 1
$$

These formulas give a piece-wise representation for the smooth alternative spectrum which today has, in all intervals of $n$, approximately the same power as the physical spectrum does.

Since the coefficients $\rho_{A_{n}}, \rho_{B_{n}}, \rho_{n}$ are fully determined, one can now derive the forms of the two power spectra at other times. We will compare the two backgrounds at the time of decoupling $\eta=\eta_{E}$. One needs to consider formulas (2.16) and (2.17), where $a(\eta)=a\left(\eta_{E}\right)$ and $y=y_{E}$,

$$
y_{E}=n\left(\eta_{E}-\eta_{m}\right)=\frac{n}{n_{d e c}}=\frac{n}{\sqrt{1+z_{d e c}}} .
$$

Clearly, in the band of sufficiently short waves, $n$ $\gg \sqrt{1+z_{d e c}}$, both spectra increase power in the same proportion, simply as a result of changing $a(\eta)$ from $a\left(\eta_{R}\right)=2 l_{H}$ to the smaller value $a\left(\eta_{E}\right)=2 l_{H} /\left(1+z_{d e c}\right)$. So, in this range of $n$ the ratio of powers in the two backgrounds is 1 :

$$
\frac{\left.h^{2}\left(n, \eta_{E}\right)\right|_{\text {alt }}}{\left.h^{2}\left(n, \eta_{E}\right)\right|_{\text {phys }}}=1, \quad n \gg \sqrt{1+z_{d e c}} .
$$

However, this ratio is significantly larger than 1 in longer waves. This is seen from the general formula

$$
\frac{\left.h^{2}\left(n, \eta_{E}\right)\right|_{\text {alt }}}{\left.h^{2}\left(n, \eta_{E}\right)\right|_{\text {phys }}}=\frac{\rho_{n}^{2}\left[j_{1}^{2}\left(n / n_{d e c}\right)+j_{-2}^{2}\left(n / n_{d e c}\right)\right]}{\left[\rho_{A_{n}} j_{1}\left(n / n_{d e c}\right)-\rho_{B_{n}} j_{-2}\left(n / n_{d e c}\right)\right]^{2}}
$$

applied to longer waves. Indeed, in the interval $1 \ll n$ $\ll \sqrt{1+z_{d e c}}$ (and, hence, $n \ll n_{m}$ ) one uses the small argu- 
ment approximation for the Bessel functions, neglects second terms in the numerator and denominator, and takes $\rho_{n}^{2}$ from Eq. (4.5). This calculation results in

$$
\frac{\left.h^{2}\left(n, \eta_{E}\right)\right|_{\text {alt }}}{\left.h^{2}\left(n, \eta_{E}\right)\right|_{p h y s}}=\frac{9}{2} \frac{\left(1+z_{d e c}\right)^{3}}{n^{6}}, \quad 1 \ll n \ll \sqrt{1+z_{d e c}} \text {. }
$$

This ratio is comparable with 1 only at $n \sim \sqrt{1+z_{d e c}}$ where Eq. (4.10) goes over into Eq. (4.8). But the ratio (4.10) is much larger than 1 for smaller $n$ 's. As for the region $n \ll 1$, one applies the same approximations as in the previous case, but takes $\rho_{n}^{2}$ from Eq. (4.6). This gives the result

$$
\frac{\left.h^{2}\left(n, \eta_{E}\right)\right|_{\text {alt }}}{\left.h^{2}\left(n, \eta_{E}\right)\right|_{\text {phys }}}=\left(1+z_{\text {dec }}\right)^{3}, \quad n \ll 1 .
$$

This ratio is universally (independently of $n$ in this region) much larger than 1 .

It is easy to understand these results. The parts of the general solution $(2.5)$ with $j_{1}(y)$ and $j_{-2}(y)$ are called, respectively, the growing and decaying solutions. This classification reflects their different behavior in the small argument approximation for the Bessel functions. The growing and decaying solutions are necessarily present in the power spectra of both backgrounds. However, the stationary background contains $j_{1}^{2}(y)$ and $j_{-2}^{2}(y)$ always with equal coefficients, whereas the nonstationary background contains $j_{1}(y)$ and $j_{-2}(y)$ with equal (up to oscillations) coefficients (4.2) only for sufficiently short waves. In longer waves, the decaying solution of the stationary background becomes progressively more important when one goes back in time. Since the physical background is expected to have enough power to produce the lower order anisotropy in the CMB temperature at the level $\delta T / T \sim 10^{-5}$, the alternative background would have produced this anisotropy at the unacceptably high level $\delta T / T \sim 3 \times 10^{-1}$.

\section{MICROWAVE BACKGROUND ANISOTROPIES CAUSED BY RELIC GRAVITATIONAL WAVES}

The key element in formula for the temperature variation $\delta T / T$ seen in a given direction $\mathbf{e}[24]$ is the $\eta$-time derivative of metric perturbations evaluated along the CMB photon's path between the event of reception (R) and the event of emission (E):

$$
\frac{\delta T}{T}(\mathbf{e})=\frac{1}{2} \int_{0}^{w_{1}}\left[\frac{\partial h_{i j}}{\partial \eta} e^{i} e^{j}\right]_{p a t h} d w .
$$

The upper limit of integration is

$$
w_{1}=\eta_{R}-\eta_{E}=1-\frac{1}{\sqrt{1+z_{d e c}}}
$$

and the integration is performed along the path $\eta=\eta_{R}-w$, $\mathbf{x}=\mathbf{e} w$. In the case of density perturbations, the integral in formula (5.1) should be augmented by the additive term rep- resenting initial conditions: an intrinsic variation of temperature at $\eta=\eta_{E}$ and a possible velocity of the last scattering electrons with respect to the chosen coordinate system, which is synchronous and comoving with the perturbed, gravitationally dominant pressureless matter, possibly, cold dark matter (CDM).

Similar to the perturbation field $h_{i j}$ itself, the temperature variation $\delta T / T$ is also a quantum-mechanical operator. To establish contact with macroscopic physics, we need to calculate the correlation function

$$
\left\langle 0\left|\frac{\delta T}{T}\left(\mathbf{e}_{1}\right) \frac{\delta T}{T}\left(\mathbf{e}_{2}\right)\right| 0\right\rangle
$$

We use the mode functions (2.5) and the normalization constant $\mathcal{C}=\sqrt{16 \pi} l_{P l}$. Then, it can be shown [22] that the correlation function takes the elegant form

$$
\left\langle 0\left|\frac{\delta T}{T}\left(\mathbf{e}_{1}\right) \frac{\delta T}{T}\left(\mathbf{e}_{2}\right)\right| 0\right\rangle=l_{P l}^{2} \sum_{l=2}^{\infty} K_{l} P_{l}(\cos \delta),
$$

where $P_{l}(\cos \delta)$ are Legendre polynomials for the separation angle $\delta$ between the unit vectors $\mathbf{e}_{1}$ and $\mathbf{e}_{2}$, and

$$
K_{l}=(2 l+1)(l-1) l(l+1)(l+2) F_{l},
$$

where

$$
F_{l}=\int_{0}^{\infty} n^{2}\left|\int_{0}^{w_{1} J_{l+1 / 2}(n w)} \frac{{ }^{2}}{(n w)^{5 / 2}} f_{n}\left(\eta_{R}-w\right) d w\right|^{d n},
$$

and

$$
f_{n}\left(\eta_{R}-w\right)=\left.\frac{1}{\sqrt{2 n}}\left(\frac{\mu_{n}}{a}\right)^{\prime}\right|_{\eta=\eta_{R}-w}
$$

We will also be using the multipole moments $C_{l}$ defined by

$$
l_{P l}^{2} K_{l}=\frac{2 l+1}{4 \pi} C_{l} .
$$

The central quantity for the calculation of $F_{l}$ is the function

$$
\left(\frac{\mu_{n}}{a}\right)^{\prime}=-\frac{n}{a} \sqrt{y}\left(A_{n} J_{5 / 2}(y)+i B_{n} J_{-5 / 2}(y)\right) .
$$

To get more insight into $F_{l}$ we introduce the two new functions: $\psi_{ \pm l}$, defined by the respective integrals:

$$
\psi_{ \pm l}\left(n w_{1}\right)=\int_{0}^{n w_{1}} d x \frac{J_{l+1 / 2}(x)}{x^{5 / 2}} \frac{J_{ \pm 5 / 2}(n-x)}{(n-x)^{3 / 2}} .
$$

Then, the general expression for $F_{l}$ takes the form

$$
\begin{aligned}
F_{l}= & \frac{1}{8 l_{H}^{2}} \int_{0}^{\infty} d n n^{5}\left[\left|A_{n}\right|^{2} \psi_{l}^{2}+\left|B_{n}\right|^{2} \psi_{-l}^{2}\right. \\
& \left.+2 \operatorname{Im}\left(A_{n}^{*} B_{n}\right) \psi_{l} \psi_{-l}\right] .
\end{aligned}
$$




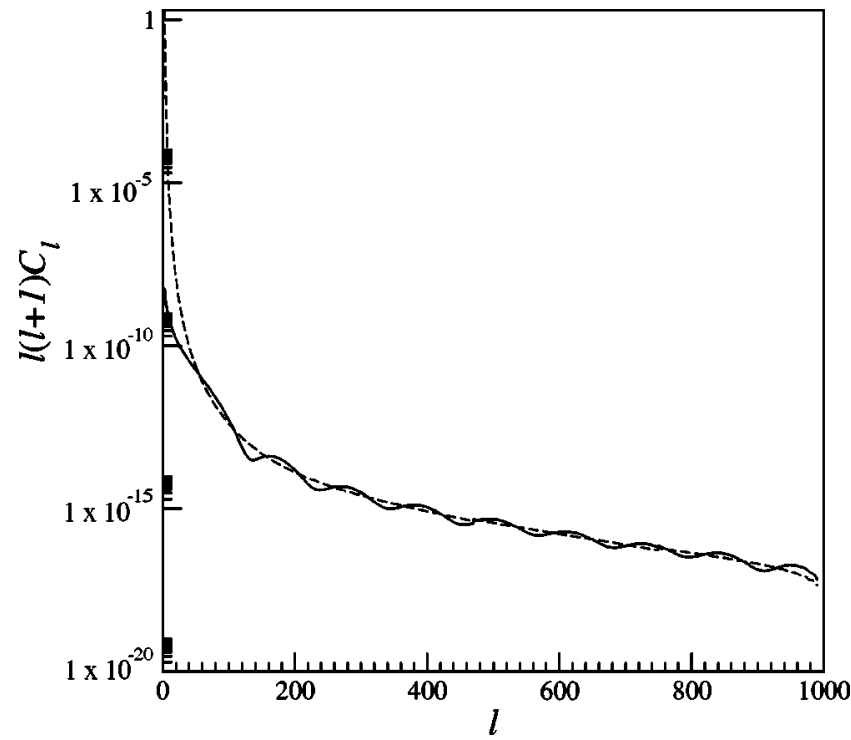

FIG. 2. The solid line depicts the plot of $l(l+1) C_{l}$ versus $l$ in the physical model, normalized such that at $l=10$, we have $l(l$ $+1) C_{l}=6.4 \times 10^{-10}$, which tallies with observations. The (red) dashed line is the corresponding plot in the alternative model. Here, we take $\beta=-2$, and the redshifts at $\eta_{2}$ and $\eta_{E}$ to be $z_{e q}=10000$ and $z_{d e c}=1000$, respectively.

This expression simplifies when the coefficients $A_{n}, B_{n}$ represent traveling [Eq. (2.12)] or standing [Eq. (2.14)] waves. For a stationary background one gets

$$
F_{l}=\frac{1}{8 l_{H}^{2}} \int_{0}^{\infty} d n n^{5} \rho_{n}^{2}\left[\psi_{l}^{2}+\psi_{-l}^{2}\right],
$$

while for the physical nonstationary background one gets

$$
F_{l}=\frac{1}{8 l_{H}^{2}} \int_{0}^{\infty} d n n^{5}\left[\rho_{A_{n}} \psi_{l}-\rho_{B_{n}} \psi_{-l}\right]^{2} \text {. }
$$

These formulas explain the different behavior of the multipole moments $C_{l}$ in the two cases. We demonstrate this with the help of numerical calculations.

In Fig. 2 we show by a solid line the graph of the function $l(l+1) C_{l}$ calculated with the help of Eqs. (5.9) and (3.12). The cosmological parameters were chosen, for illustration, as $z_{e q}=10^{4}, z_{d e c}=10^{3}, \beta=-2$. The parameter $z_{i}$ is adjusted in such a manner $\left(z_{i}=10^{29.5}\right)$ that the graph goes through the point $l(l+1) C_{l}=6.4 \times 10^{-10}$ at $l=10$, which agrees with observations. Our attention is focused, however, on the oscillations in this function. The dashed line shows the same function for the alternative stationary background. The cosmological parameters are the same as above, but the calculation is performed with the help of Eq. (5.8) and the coefficients $\rho_{n}$, found from the condition (4.1) of the fair comparison. The remarkable (even if expected) result is that the stationary background of gravitational waves does not produce oscillations in the angular power spectrum $C_{l}$, whereas the nonstationary background does.

The numerical positions of minima and maxima in the oscillating graph are ordered as follows:

$$
\begin{array}{lll}
\text { Minima: } & l_{1}=137, \quad l_{2}=237, \quad l_{3}=344, \quad l_{4}=456, \\
& l_{5}=569, \quad l_{6}=682, \quad l_{7}=796, \\
\text { Maxima: } & l_{1}=161, \quad l_{2}=269, \quad l_{3}=381, \quad l_{4}=494, \\
& l_{5}=609, \quad l_{6}=723, \quad l_{7}=839 .
\end{array}
$$

Clearly, these features reflect the oscillations in the metric power spectrum. Judging from the mathematical structure of the participating Bessel functions, it is likely that the positions of features in the $n$ space are related to the positions of features in the $l$-space by a simple numerical coefficient of order 1. It is difficult to find out this coefficient analytically, though. Remembering that the positions of first features may be displaced by $10 \%-15 \%$, as compared with the analytical forecast (3.19) and (3.18), we put the zeroth "would be" maximum at $l_{0}^{\max }=56$. Then, our simple analytical formula places the next features in the following positions: $l_{1}^{\text {min }}$ $=112, l_{1}^{\max }=168, l_{2}^{\min }=224, l_{2}^{\max }=280, l_{3}^{\min }=336, l_{3}^{\max }$ $=392, l_{4}^{\text {min }}=448, l_{4}^{\text {max }}=504, l_{5}^{\text {min }}=560, l_{5}^{\text {max }}=616, l_{6}^{\text {min }}$ $=672, l_{6}^{\max }=728, l_{7}^{\min }=784, l_{7}^{\max }=840$. Comparing this prediction with the numerically calculated positions, we find them in a fairly good agreement.

This investigation of gravitational waves provides us with guidance for the technically more complicated case of density perturbations.

\section{DENSITY PERTURBATIONS AND THE $C_{\ell}$ FEATURES}

The general expression for metric perturbations, associated with density perturbations, is given by Eq. (1.3) with the polarization tensors explained in the Introduction. When one is actually writing down the perturbed Einstein equations, it turns out that it is more convenient to work with the mode functions $h(\eta)$ and $h_{l}(\eta)$ instead of the original mode functions $h_{n}(\eta)$. The relationship between them is

$$
\stackrel{1}{h}_{n}(\eta)=\sqrt{\frac{3}{2}}\left(h(\eta)-\frac{1}{3} h_{l}(\eta)\right), \quad \stackrel{2}{h}_{n}(\eta)=\frac{1}{\sqrt{3}} h_{l}(\eta)
$$

where the wave-number index $n$ on the mode functions $h(\eta)$ and $h_{l}(\eta)$ is implicit. The function $h(\eta)$ is the purely scalar part of perturbations, it enters $h_{i j}$ with the polarization structure $\stackrel{1}{P}_{i j}=\delta_{i j}$, whereas the function $h_{l}(\eta)$ is the purely longitudinal-longitudinal part of perturbations, it enters $h_{i j}$ with the polarization structure $\stackrel{2}{P}_{i j}=-n_{i} n_{j} / n^{2}$. We will follow the same strategy as in the case of gravitational waves, and will start from exact solutions to the perturbed Einstein equations in different cosmological eras.

\section{A. Density perturbations at the matter-dominated stage}

The matter-dominated stage is driven by a pressureless matter; possibly, cold dark matter. The general solution to the perturbed equations at the $m$ stage can be simpli- 
fied by using the available freedom within the class of synchronous coordinate systems. By using this freedom, one specializes to the unique coordinate system, which is synchronous and comoving with the perturbed pressureless matter. In this coordinate system, the general solution is

$$
\begin{aligned}
h(\eta) & =C_{1}, \\
h_{l}(\eta) & =\frac{1}{10} C_{1} n^{2}\left(\eta-\eta_{m}\right)^{2}-\frac{1}{3} C_{2} \frac{\left(\eta_{2}-\eta_{m}\right)^{3}}{\left(\eta-\eta_{m}\right)^{3}},
\end{aligned}
$$

where $C_{1}, C_{2}$ are arbitrary complex numbers. The matter density perturbation is

$$
\frac{\delta \epsilon}{\epsilon_{0}}=\frac{1}{2} h_{l}(\eta),
$$

and the velocity $v^{j}$ of matter elements, including perturbations,

$$
\frac{v^{j}}{c}=\frac{T_{0}^{j}}{T_{0}^{0}}
$$

is by construction zero, i.e.,

$$
\frac{v^{j}}{c}=0
$$

The above solution is well known since the times of E.M. Lifshitz and can be found in various publications, up to possible misprints. For instance, this solution follows from equations given in Ref. [25], if one corrects for a misprint in the last line of Eq. (A8): the second term there should actually enter with coefficient 2 .

The adopted choice of the unique coordinate system, which is both comoving and synchronous, not only greatly simplifies the form of solutions, but is also needed for a proper formulation of the $\delta T / T$ calculations. As long as the emitter and the receiver are riding on the time-like geodesics $x^{i}=$ const of this perturbed metric, the Sachs-Wolfe integral (5.1) is the full answer; there are no extra velocity contributions to this integral. The additive velocity contributions arise only if the emitter or the receiver are moving with respect to this unique coordinate system, that is, when they are not described by the world-lines $x^{i}=$ const.

For the growing solution (namely, the terms with coefficient $C_{1}$ ), the Sachs-Wolfe integral can be taken exactly. It appears that astrophysical literature calls by gravitational "Sachs-Wolfe effect" only a part of what is actually contained in the Sachs-Wolfe paper [24]. Invariably, by the "Sachs-Wolfe effect" are meant only two terms, which are, roughly speaking, the difference of "gravitational potentials" at the events of emission (E) and reception (R). Two other terms in their full formula (43), which are the difference of the "gradients of the gravitational potential," are being systematically ignored. Possibly, this happened because Sachs and Wolfe addressed one of these gradient terms in the words: "this second term is normally small." This second term is indeed small for small wave numbers, but it is in fact dominant for large wave numbers, which are responsible for the dipole $C_{1}$ and for the $C_{l}$ multipoles near the peak at $l$ $\sim 200$. For example, correct implementation of the full Sachs-Wolfe formula (43) for calculation of the dipole $C_{1}$ gives a number that is five orders of magnitude greater than the number following from the "Sachs-Wolfe effect" counterpart of the full formula. The lack of ergodicity on a 2 -sphere provides a $1 \sigma$ uncertainty in the $C_{l}$ 's, roughly at the level $\Delta C_{l} \simeq \sqrt{2 /(2 l+1)} C_{l}$. We say "roughly" because the statistic of the underlying random variable is not Gaussian, it is described by the product of an exponent and the modified Bessel function $K_{0}$ [14]. In the case of the dipole $C_{1}$, the uncertainty amounts to $\Delta C_{1} / C_{1} \approx 0.8$. Clearly, this factor-oftwo uncertainty cannot cover the five orders of magnitude disparity in the results; quite simply, the result based on the misinterpreted "Sachs-Wolfe effect" is what is wrong. For details, see Ref. [15].

The coefficients $C_{1}, C_{2}$ in the general solution (6.2) are, so far, arbitrary, but they are determined by the previous evolution of density perturbations (dp).

\section{B. Density perturbations at the radiation-dominated stage}

The "master equation" at the $e$ stage is

$$
\nu^{\prime \prime}+\frac{1}{3} n^{2} \nu=0,
$$

where the coefficient $1 / 3$ enters because we have used $c_{l} / c$ $=1 / \sqrt{3}$, which is valid deep in the radiation-dominated era. By the time of decoupling, the plasma sound speed decreases slightly below this value, depending on the baryon content, and we will account for this fact by returning back to $c_{l} / c$ in appropriate places. The general solution to Eq. (6.6) is always oscillatory as a function of time:

$$
\nu=B_{1} e^{-i(n / \sqrt{3})\left(\eta-\eta_{e}\right)}+B_{2} e^{i(n / \sqrt{3})\left(\eta-\eta_{e}\right)},
$$

where $B_{1}, B_{2}$ are arbitrary complex numbers. All the metric and matter perturbations can now be found from solutions (6.7). For metric perturbations, one has:

$$
h(\eta)=\frac{a^{\prime}}{a^{2}}\left[\int_{\eta_{1}}^{\eta} \nu \mathrm{d} \eta+C_{e}\right]
$$

and

$$
h_{l}^{\prime}=\frac{a}{a^{\prime}}\left[3 h^{\prime \prime}+9 \frac{a^{\prime}}{a} h^{\prime}+n^{2} h\right] .
$$

The constant $C_{e}$ reflects the remaining coordinate freedom at the $e$ stage. The $C_{e}$ should be chosen in such a way that the comoving synchronous coordinate system of the $m$ stage joins smoothly to the employed (unique) coordinate system at the $e$ stage; we will discuss this specific choice of $C_{e}$ later on. The constants $B_{1}, B_{2}$ are still arbitrary and should be found from solutions at the $i$ stage.

The "master equation" at the $i$ stage is 


$$
\mu_{n}^{\prime \prime}+\mu_{n}\left[n^{2}-\frac{(a \sqrt{\gamma})^{\prime \prime}}{a \sqrt{\gamma}}\right]=0
$$

where

$$
\gamma \equiv 1+\left(\frac{a}{a^{\prime}}\right)^{\prime} \equiv-\frac{\dot{H}}{H^{2}}
$$

and the $t$-time derivative is related with the $\eta$-time derivative by $c \mathrm{~d} t=a \mathrm{~d} \eta$. For the power-law scale factors $a(\eta)$ $\propto|\eta|^{1+\beta}$, which we are working with, the function $\gamma$ becomes a constant, and it drops out of the Eq. (6.10). So, the "master equation" (6.10) is exactly the same as Eq. (2.1) for gravitational waves. By quantum-normalizing the initial metric perturbations, and evolving them through the $i$ stage, we finally find that

$$
B_{1} \approx-B_{2} \equiv B_{\mathrm{dp}} .
$$

It was shown [11] that the crucial quantity $B_{\mathrm{dp}}$ for density perturbations is related with the crucial quantity $B_{\mathrm{gw}}$ for gravitational waves, introduced in Eq. (3.9), by the relationship

$$
B_{\mathrm{dp}}=\sqrt{6} B_{\mathrm{gw}} .
$$

In what follows, we will work with $B_{\mathrm{dp}}$ only and, henceforth, drop the subscript dp.

Combining all the results together, we write down explicitly the exact solution at the $e$ stage, including the required choice of $C_{e}$. In doing this, we use the following new notations:

$$
\begin{aligned}
y & \equiv \frac{n}{\sqrt{3}}\left(\eta-\eta_{e}\right), \\
y_{2} & \equiv \frac{n}{\sqrt{3}}\left(\eta_{2}-\eta_{e}\right)=\frac{n}{\sqrt{3} 2 \sqrt{1+z_{e q}}}=\frac{n}{n_{c}}, \\
n_{c} & \equiv 2 \sqrt{3} \sqrt{1+z_{e q}}, \quad Y \equiv \frac{1}{2} y_{2} \sin y_{2}+\cos y_{2} .
\end{aligned}
$$

Then, the exact solution is

$$
\begin{aligned}
h(\eta)= & \frac{A}{y^{2}}[\cos y-Y], \\
h_{l}(\eta)= & 3 A\left[-\frac{\sin y}{y}-\int_{y}^{y_{2}} \frac{\cos y}{y} \mathrm{~d} y-Y \ln \frac{y}{y_{2}}\right. \\
& \left.+\frac{1}{3} \frac{\sin y_{2}}{y_{2}}+\frac{2}{3} \cos y_{2}\right], \\
\frac{\delta \epsilon}{\epsilon_{0}}= & -A\left[\frac{2}{y^{2}}(\cos y-Y)+\frac{2}{y} \sin y-\cos y\right],
\end{aligned}
$$

$$
\frac{v^{j}}{c}=-i A \frac{n^{j}}{n \sqrt{3}}\left[\frac{2}{y}(\cos y-Y)+\sin y\right],
$$

where

$$
A \equiv \frac{i n B \sqrt{1+z_{e q}}}{2 \sqrt{3} l_{H}} .
$$

One can check that all the participating functions, $h(\eta), h^{\prime}(\eta), h_{l}(\eta), h_{l}^{\prime}(\eta), \delta \epsilon / \epsilon_{0}, v^{j}$, join continuously with the solution $(6.2),(6.3),(6.5)$ at the transition point $\eta=\eta_{2}$. This transition fully determines the coefficients $C_{1}$ and $C_{2}$ :

$$
\begin{aligned}
& C_{1}=-\frac{A}{2 y_{2}} \sin y_{2}, \\
& C_{2}=\frac{3 A}{5 y_{2}}\left[\left(10-3 y_{2}^{2}\right) \sin y_{2}-10 y_{2} \cos y_{2}\right] .
\end{aligned}
$$

The oscillatory behavior of $C_{1}, C_{2}$, as functions of $n$, is analogous to the oscillatory behavior of the gravitational wave coefficients (3.12) and has the same physical origin. The fact that $B_{1} \approx-B_{2}$ demonstrates that each mode $\mathbf{n}$ of the metric perturbations, and the associated matter perturbations, at the $e$ stage forms a standing wave pattern. In the limit of short waves, $y \gg 1$, one recovers from Eqs. (6.17) and (6.18) the familiar solutions for standing sound waves:

$$
\begin{aligned}
& \frac{\delta \epsilon}{\epsilon_{0}} \approx A \cos y, \\
& \frac{v^{j}}{c} \approx-i A \frac{n^{j}}{n \sqrt{3}} \sin y .
\end{aligned}
$$

\section{Perturbations at the last scattering surface}

Having found the quantum-normalized exact solution at the $m$ stage, we are in a position to calculate the metric power spectrum, which is defined by Eq. (1.7). Taking into account our mode functions, the spectrum can be written as

$$
h^{2}(n, \eta)=\frac{\mathcal{C}^{2}}{2 \pi^{2}} n^{2}\left[\frac{3}{2}\left|h-\frac{1}{3} h_{l}\right|^{2}+\frac{1}{3}\left|h_{l}\right|^{2}\right] .
$$

We will calculate this quantity at the last scattering surface $\eta=\eta_{E}$. By that time, the second term in the function $h_{l}(\eta)$ is a factor $\left[\left(1+z_{d e c}\right) /\left(1+z_{e q}\right)\right]^{5 / 2}$ smaller than the first term [see Eqs. (6.2) and (6.20)]. We neglect this decaying part of the solution, participating with the coefficient $C_{2}$. For the explicit form of $\left|C_{1}\right|^{2}$ we use Eqs. (6.20) and (6.19). Then, we obtain

$$
\begin{aligned}
h^{2}\left(n, \eta_{E}\right)= & \frac{\mathcal{C}^{2}}{2 \pi^{2}} \frac{n^{4}|B|^{2}\left(1+z_{e q}\right)}{48 l_{H}^{2}}\left(\frac{\sin y_{2}}{y_{2}}\right)^{2} \\
& \times \frac{\left(300-20 p^{2} y_{2}^{2}+p^{4} y_{2}^{4}\right)}{200},
\end{aligned}
$$




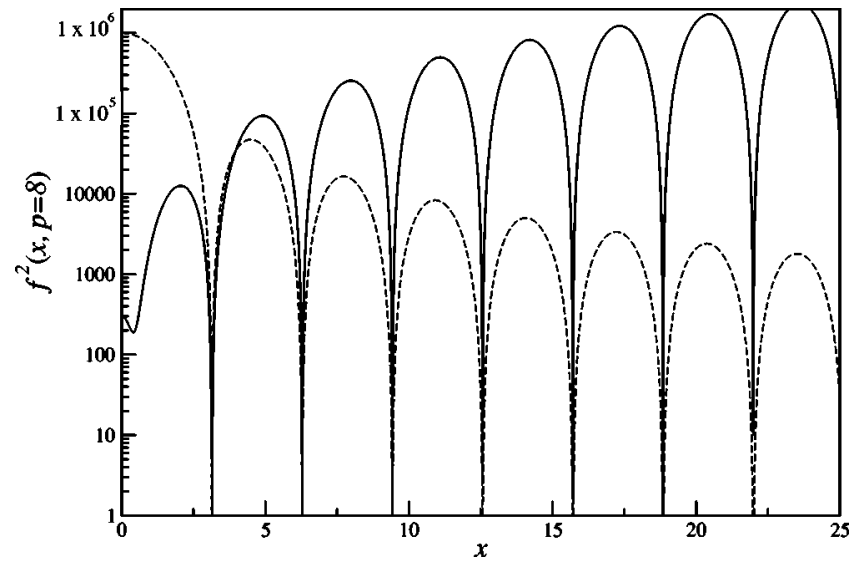

FIG. 3. The plot depicted by the solid line is that of $f^{2}(x, p$ $=8)$ vs $x$. The dashed line shows the behavior of $M^{2}(x) \times 10^{6}$.

where we have introduced the quantity

$$
p \equiv \frac{2 \sqrt{3} \sqrt{1+z_{e q}}}{\sqrt{1+z_{d e c}}},
$$

related to a similar quantity, $b$, from the gw case, by $p$ $=\sqrt{3} b$. The spectrum certainly retains its primordial form in the band of long waves $n \ll \sqrt{1+z_{d e c}}$. Taking into account Eq. (6.12) and the numerical value of $\mathcal{C}=\sqrt{24 \pi} l_{P l}$ for density perturbations, one finds that the primordial spectrum of metric perturbations associated with density perturbations, Eq. (6.24), is a factor of 9/16 lower than its gravitational wave counterpart, Eq. (3.15). In particular, for $\beta=-2$, one finds

$$
h^{2}\left(n, \eta_{E}\right) \approx \frac{9}{\pi} \frac{l_{P l}^{2}}{l_{H}^{2}} \frac{\left(1+z_{i}\right)^{4}}{\left(1+z_{e q}\right)}, \quad n \ll n_{d e c}=\sqrt{1+z_{d e c}}
$$

For relatively short waves, $n / n_{c} \gg 1$, the crucial part of the power spectrum (6.24) is the modulating (transfer) function

$$
M^{2}\left(\frac{n}{n_{c}}\right)=\left(\frac{\sin y_{2}}{y_{2}}\right)^{2}=\frac{\sin ^{2}\left(n / n_{c}\right)}{\left(n / n_{c}\right)^{2}} .
$$

The primordial metric spectrum is encoded in the factor $n^{4}|B|^{2}$. Whatever this spectrum is, the modulating function leaves it intact at large scales, but bends the spectrum down and introduces oscillations at smaller scales. In Fig. 3 we show the metric power spectrum $h^{2}\left(n, \eta_{E}\right)$, up to numerical coefficients. [To avoid confusion, we emphasize again that this is the spectrum of the (squeezed) metric perturbations associated with density perturbations, and not the gravitational-wave spectrum [26].] Specifically, by a solid line, we plot the function $f^{2}(x, p)$, where

$$
f^{2}(x, p) \equiv\left(\frac{\sin x}{x}\right)^{2}\left[300-20 p^{2} x^{2}+p^{4} x^{4}\right],
$$

$x \equiv y_{2}=n / n_{c}$ and, for illustration, we take $p=8$. By a dashed line, we plot the function $M^{2}(x)$, multiplied by the artificial numerical factor $10^{6}$ in order to facilitate the visual comparison of maxima and zeros in the two graphs.

We now turn to the ordinary matter perturbations at the last scattering surface. The photon-electron-baryon fluid is gravitationally subdominant at $\eta=\eta_{E}$. The fluid does not significantly contribute to metric perturbations, but it retains its own perturbations. The plasma speed of sound is given by

$$
\frac{c_{l}}{c}=\frac{1}{\sqrt{3(1+R)}}
$$

where $R=3 \rho_{b} / 4 \rho_{\gamma} \approx 27 \Omega_{b} h^{2}[19,20]$. For the popular value $\Omega_{b} h^{2} \approx 0.02$, it means that $c_{l} / c$ decreases from the nominal value 0.58 to approximately 0.47 . The plasma standing waves (6.21) and (6.22), continued to the decoupling era $\eta$ $=\eta_{E}$, take the form:

$$
\begin{aligned}
\frac{\delta \epsilon_{\gamma}}{\epsilon_{\gamma}} & \approx A \cos \frac{n}{n_{s}}, \\
\frac{v^{j}}{c} & \approx-i A \frac{c_{l}}{c} \frac{n^{j}}{n} \sin \frac{n}{n_{s}},
\end{aligned}
$$

where

$$
n_{s}=\frac{c}{c_{l}} \sqrt{1+z_{d e c}} \equiv \frac{c}{c_{l}} n_{d e c}
$$

[The velocity $v^{j}$ is always defined with respect to the unique synchronous coordinate system, which is comoving with the gravitationally dominant pressureless (dark) matter.] At the same time, the leading metric perturbation is given by

$$
h_{l}\left(\eta_{E}\right)=-\frac{3}{5} A \frac{1+z_{e q}}{1+z_{d e c}} \frac{n}{n_{c}} \sin \frac{n}{n_{c}} .
$$

There are a number of differences between the metric perturbations and the plasma perturbations at the last scattering surface. First, the amplitude of $h_{l}\left(\eta_{E}\right)$ is, at least, a factor of $3\left(1+z_{e q}\right) /\left[5\left(1+z_{\text {dec }}\right)\right]$ greater than the amplitudes of $\delta \epsilon_{\gamma} / \epsilon_{\gamma}$ and $v^{j} / c$, near the most interesting scales $n \approx n_{c}$. After all, the original motivation for the introduction of a cosmological dark matter was precisely this: to avoid conflicts with $\delta T / T$ observations by allowing the plasma perturbations at decoupling to be small, but, nevertheless, to be able to develop the large scale structure of luminous matter, at the expense of large gravitational field perturbations driven by the dark matter. So, we have to pay the price for this idea by exploring in more detail the consequences of large metric perturbations for the CMB anisotropies.

Second, the characteristic frequencies $n_{c}$ and $n_{s}$ are different. Their ratio is

$$
\frac{n_{c}}{n_{s}}=2 \sqrt{3} \frac{c_{l}}{c} \sqrt{\frac{1+z_{e q}}{1+z_{d e c}}} .
$$

The $z_{e q}$ is given by $1+z_{e q} \approx 4 \times 10^{4} \Omega_{m} h^{2}$ [19]. For the popular values $\Omega_{m}=0.3, h=0.7$, this amounts to $z_{e q} \approx 6$ $\times 10^{3}$. So, the ratio $n_{c} / n_{s}$ can be a number close to 4 . 
Third, although the sound waves before the decoupling are standing waves, they are still not processed by the quick drop of the sound speed to zero at the decoupling. This processing will later lead to the baryonic matter power spectrum modulations known as the Sakharov oscillations [27]. They would have taken place even in laboratory conditions, where gravity plays no role. The Sakharov oscillations are important for the formation of oscillating features in the luminous matter power spectrum, but they are unlikely to be directly responsible for the peaks and dips in the observed $C_{l}$ 's. In a broad sense, the periodicity in the metric power spectrum, related to the transition $\eta=\eta_{2}$, can also be called Sakharov oscillations, but this is not what was originally meant by the Sakharov oscillations. In short, the zeros in the metric power spectrum are "frozen" zeros, they are determined by $M^{2}\left(n / n_{c}\right)$; whereas the zeros in the plasma power spectrum, at the times before decoupling, are still "moving" zeros; they change their positions at slightly different moments of time $\eta=$ const [28].

Fourth, the wave-number periodicity in the metric power spectrum is governed by the sine function, whereas the periodicity in Eq. (6.28) is governed by the cosine function. Presently, there exists a tendency to distinguish between the "acoustic peaks" in the $C_{l}$ [supposedly caused by Eq. (6.28) and by the "effective temperature"] and the "Doppler peaks" [supposedly caused by the velocity in Eq. (6.29)]. The authors of [29] emphasize that "the acoustic peaks are not Doppler peaks," arguing that the irrotational velocity cannot produce strong peak structures in the $C_{l}$ spectrum. They say that "the observed peaks must be acoustic peaks" and they give the ratio of the peak locations: $\ell_{1}: \ell_{2}: \ell_{3}$ $\sim 1: 2: 3$. So, the main contenders for the explanation of the peaks seem to be the sine function in the metric power spectrum and the cosine function in the "acoustic peaks."

Before proceeding to the discussion of peaks and dips, we need to make one more comment. It was shown above that the primordial power spectra of gravitational waves and density perturbations are of the same order of magnitude, with some small numerical preference for gravitational waves. In particular, this is true for the flat spectra $(\beta=-2)$, as demonstrated in Eqs. (3.16) and (6.25). Therefore, the lower order CMBR anisotropies (starting from the quadrupole moment $C_{2}$ ) are expected to be of the same order of magnitude [11]. One should be aware that the story is dramatically different in an inflationary scenario. The "standard result" of an inflationary scenario [30-37] predicts the infinitely large density perturbations, in the limit of the flat spectrum (that is, the Harrison-Zel'dovich-Peebles spectrum, with spectral index $n=1$, parameter $\beta=-2$, and the relationship between them being $n=2 \beta+5)$, through the set of evaluations: $\delta \rho / \rho \sim h_{S} \sim H^{2} / \dot{\varphi} \sim V^{3 / 2}(\varphi) / V^{\prime}(\varphi) \sim 1 / \sqrt{1-n}$. By composing the ratio of the gravitational wave amplitude $h_{T}$ to the predicted divergent amplitude of the scalar metric perturbations $h_{S}$ (the so-called "consistency relation:" $h_{T} / h_{S}$ $\approx \sqrt{1-n}$ ), inflationary theorists substitute their prediction of arbitrarily large density perturbations for the claim that it is the amount of gravitational waves that should be zero, or almost zero, at cosmological scales and, hence, down to laboratory scales. This claim has led to many years of mistreatment of a possible gw contribution to the CMBR data. It is only in a few recent papers (for example, [38]) that the inflationary "consistency relation" is not being used when analyzing the CMBR and large scale structure observations, with some interesting conclusions. For the latest statement that the initial spectrum of gravitational waves is "constrained to be small compared with the initial density spectrum" see the latest article praising inflationary predictions (for instance, [29]). For the critical analysis of the "standard inflationary result" see the end of Sec. VI in [3] and references therein.

\section{Peaks and dips in the angular power spectrum}

We will now analyze the zeros and maxima of the metric power spectrum $f^{2}(x, p)$ shown in Fig. 3. The crucial periodic dependence is provided by the function $\sin ^{2}(x)$ $\equiv \sin ^{2}\left(n / n_{c}\right)$. We will use this function for our analytical evaluation, in full analogy with the case of gravitational waves. The positions of maxima and zeros are determined by the rules:

$$
\begin{aligned}
\text { Maxima: } x_{k}^{\text {max }} & =\frac{\pi}{2}(2 k+1), \\
x_{k}^{\text {max }} & =x_{0}^{\max }(2 k+1), \quad k=0,1,2,3, \ldots, \\
\text { Zeros: } \quad x_{k}^{\text {min }} & =\frac{\pi}{2} 2 k, \\
x_{k}^{\text {min }} & =x_{1}^{\text {min }} k, \quad k=1,2,3, \ldots .
\end{aligned}
$$

Obviously, the zeros of the function $\sin ^{2}(x)$ are exactly the same as the zeros of the full function $f^{2}(x, p)$. But the positions of maxima are somewhat different. The difference is significant for the zeroth maximum, but it fully disappears for later maxima. The locations of the first few maxima, derived from the simple analytical formula (6.33), are $x_{0}^{\max }$ $=1.57, x_{1}^{\max }=4.71, x_{2}^{\max }=7.85, x_{3}^{\max }=11.00$. At the same time, accurate positions from the numerical calculation are $x_{0}^{\max }=2.05, x_{1}^{\max }=4.92, x_{2}^{\max }=7.98, x_{3}^{\max }=11.09$. Thus formula (6.33) predicts the positions of the first two maxima somewhat to the left ( $\operatorname{smaller} x$ ) than they should actually appear, but the positions of zeros and further maxima are described very well. In terms of the percentage corrections, the zeroth maximum, derived from Eq. (6.33), should be shifted to the right by $30 \%$, and the first maximum should be shifted to the right by $4 \%$.

Accepting $z_{e q}=6 \times 10^{3}$, one obtains $n_{c}=268$. With this $n_{c}$ and $x_{0}^{\max }=1.57$, the position of the zeroth maximum in the $n$ space would be, according to Eq. (6.33), at $n_{0}^{\max }$ $=421$. Positions of all the subsequent features in the power spectrum follow from the general rules (6.34) and (6.33). The problem now is to relate these features in the metric power spectrum with the peaks and dips in the angular power spectrum $l(l+1) C_{l}$. Judging from the previous numerical experience [15], the characteristic features of the metric power spectrum are reflected in the $l$-space via a numerical 
coefficient $\alpha$ close to $1 / 2: l=\alpha n$. Accepting the provisional value $\alpha \approx 1 / 2$, the location of the zeroth peak in the $l$ space would be near $l_{0}=210$. This is a satisfactory intermediate result, but we want to do better. Remembering that the position of the zeroth peak, following from the analytical formula (6.33), should be shifted to the larger values of $l$, we place our zeroth peak at $l_{0}^{\max }=170$. The $30 \%$ correction of this number shifts the zeroth peak to $l_{0}^{\max }=221$. Of course, we keep an eye on the actually detected peak in this region. Our aim is to derive the full structure of peaks and dips in the angular power spectrum $l(l+1) C_{l}$ from the simple analytical formulas (6.33) and (6.34), allowing only for the 30\% correction to the zeroth peak and the $4 \%$ correction to the first peak. Following this strategy, we formulate our full forecast:

$$
\begin{array}{ll}
\text { Peaks: } & l_{0}^{\text {max }}=170(221), \quad l_{1}^{\text {max }}=510(530), \\
& l_{2}^{\text {max }}=850, \quad l_{3}^{\text {max }}=1190, \quad l_{4}^{\text {max }}=1530, \\
\text { Dips: } \quad & l_{1}^{\text {min }}=340, \quad l_{2}^{\text {min }}=680, \quad l_{3}^{\text {min }}=1020, \\
& l_{4}^{\text {min }}=1360, \quad l_{5}^{\text {min }}=1700 .
\end{array}
$$

As a consequence of Eqs. (6.33) and (6.34), the general rule for the peak positions is $1: 3: 5: 7 \ldots$, for the dip positions: $1: 2: 3: 4 \ldots$, and the dips appear between the peaks at

$$
l_{k}^{\min }=\frac{1}{2}\left(l_{k}^{\max }+l_{k-1}^{\max }\right)
$$

Everywhere in this paper, both for gravitational waves and density perturbations, we perform calculations under the simplifying assumption that the Universe is spatially flat. It is obvious, however, that neither the generating mechanism itself nor the results, for wavelengths comfortably shorter than the putative curvature radius, depend on this simplification. The unaccounted factors, such as the possible presence of a spatial curvature, or a $\Lambda$ term, or a "quintessence," or a "dark energy," can move the entire structure of peaks and dips, but these factors can hardly change the general rules for their relative positions.

One should note that what is following from our classification as the "zeroth gravitational peak," which we place at $l_{0}^{\max }=170$ plus the correction shifting it to $l_{0}^{\max }=221$, is often interpreted as the "first Doppler peak" or the "first acoustic peak." The notion of the "zeroth Doppler peak" was introduced and discussed by Weinberg $[19,20]$. In general, all three sources: gravitational field perturbations, intrinsic temperature variations, and velocities, contribute to the peak structure. The gravitational field contribution is represented by the Sachs-Wolfe integral (5.1), while the two other sources are represented by Eqs. (6.28) and (6.29). However, the raising function $l(l+1) C_{l}$ would not have turned down without the modulating function $M^{2}\left(n / n_{c}\right)$ [15], so we focus our attention on the gravitational contribution.
The numerical graph of Fig. 3 also shows a little depression at $x_{d e p}=0.41$. This depression arises entirely due to the polynomial term in $f^{2}(x, p)$ rather than from the modulating function $M^{2}(x)$. Accepting the same value $n_{c}=268$, this feature corresponds to $n_{d e p}=110$. Assigning some significance to this feature, and following the same logic as before, we have to conclude that this depression in the metric power spectrum may be reflected as a small local minimum in the angular power spectrum. Applying the numerical factor $\alpha$ $=1 / 2$, this minimum is expected to be seen around $l_{d e p}$ $\approx 55$. This may be one of the areas in the $l$ space to analyze closely in future experiments, such as the Microwave Anisotropy Probe (MAP) and Planck.

To compare our forecast with observations, we take for the face value the central positions of peaks and dips reported by de Bernardis et al. [17]. We take the liberty of calling their Peak 1 as our zeroth peak, Peak 2 as the first peak, and so on. The reported measured positions are as follows:

$$
\begin{array}{ll}
\text { Peaks: } & l_{0}^{\max }=213, \quad l_{1}^{\max }=541, \quad l_{2}^{\max }=845, \\
\text { Dips: } & l_{1}^{\min }=416, \quad l_{2}^{\min }=750 .
\end{array}
$$

Their forecast for the next features is as follows:

$$
\begin{aligned}
\text { Peaks: } & l_{3}^{\max }=1139, \quad l_{4}^{\max }=1442, \\
\text { Dips: } & l_{3}^{\text {min }}=1025, \quad l_{4}^{\min }=1328, \quad l_{5}^{\min }=1661 .
\end{aligned}
$$

Comparing the observed positions (6.36) with our formulas (6.35), we find them in reasonably good agreement. The peaks and dips appear, at least roughly, in the right positions. On the other hand, the periodic function $\cos ^{2}\left(n / n_{s}\right)$, appropriate for the "acoustic peaks," implies the reversed rules for the dip and peak positions:

$$
\begin{aligned}
& \text { Dips: } l_{k}^{\text {min }}=l_{0}^{\min }(2 k+1), \quad k=0,1,2,3, \ldots ; \\
& \text { Peaks: } \quad l_{k}^{\text {max }}=l_{1}^{\max } k, \quad k=1,2,3, \ldots .
\end{aligned}
$$

So, the structure is supposed to start from the zeroth dip, the dip positions are ordered as $1: 3: 5: 7 \ldots$, the peak positions are ordered as $1: 2: 3: 4 \ldots$, and the peaks appear between neighboring dips at $l_{k}^{\max }=(1 / 2)\left(l_{k}^{\min }+l_{k-1}^{\min }\right)$. If the first acoustic peak is at $l \approx 213$, the second one is supposed to be at $l \approx 426$, almost in the same place where observations indicate the first dip. Most importantly, there is no observational evidence whatsoever for the zeroth dip. We do not see how the observed structure (6.36) can be explained by the acoustic peaks.

The forecast (6.35) on one side, and the forecast (6.37) on the other side, go out of phase at late features. We place our fourth peak in between the positions where de Bernardis et al. [17] place their $l_{4}^{\max }$ and $l_{5}^{\min }$. If these features are 
not washed out by damping $[19,20,29]$ the MAP and Planck missions will provide the answer. So far, we tentatively conclude that the structures in the angular power spectrum are caused by squeezing in the primordial gravitational field perturbations associated with the density perturbations.

Note added in proof. The latest Cosmic Background Imager observations [39] have detected four peaks, at $l \sim 550$, 800, 1150, 1500, and four dips, at $l \sim 400,700,1050,1400$. These positions are in very good agreement with the theoretical formula (6.35) of the present paper. We interpret these data as confirmation of our conclusion that it is gravity, and not acoustics, that is responsible for the observed structure.

\section{ACKNOWLEDGMENTS}

We would like to thank P. de Bernardis and P. Mauskopf for a helpful discussion on peaks and dips in the angular power spectrum. Thanks are also due to Bruce Allen, B.S. Sathyaprakash, and Massimo Tinto for useful discussions. S.B. thanks S. Koranda for providing the numerical code of Ref. [23] and for showing us how to implement it in order to reproduce the results given in that reference. He also thanks A. Dimitropoulos for interesting discussions. L.P.G. would like to thank K. Glampedakis for help in graphing some of the equations discussed here. The work of S.B. was funded in part by PPARC grant no. PPA/G/S/1997/00276.
[1] K. S. Thorne, in 300 Years of Gravitation, edited by S. Hawking and W. Israel (Cambridge University Press, Cambridge, England, 1987); in Particle and Nuclear Astrophysics and Cosmology in the Next Millennium, Snowmass 94, edited by E. W. Kolb and R. D. Peccei (World Scientific, River Edge, 1995), gr-qc/9506086.

[2] B. F. Schutz, Class. Quantum Grav. 16, A131 (1999).

[3] L. P. Grishchuk, V. M. Lipunov, K. A. Postnov, M. E. Prokhorov, and B. S. Sathyaprakash, Usp. Fiz. Nauk. 171, 3 (2001) [Phys. Usp. 44, 1 (2001)].

[4] L. P. Grishchuk, Zh. Éksp. Teor. Fiz. 67, 825 (1974) [JETP 40, 409 (1975)]; Ann. N.Y. Acad. Sci. 302, 439 (1977).

[5] E. Schrödinger, Physica (Amsterdam) 6, 899 (1939).

[6] L. P. Grishchuk, Phys. Rev. D 48, 5581 (1993).

[7] L. Parker, Ph.D. thesis, Harvard University, 1966; Phys. Rev. Lett. 21, 562 (1968); Phys. Rev. 183, 1057 (1969); Phys. Rev. D 3, 346 (1971).

[8] A. A. Grib, S. G. Mamaev, and V. M. Mostepanenko, Quantum Effects in Intense External Fields (Atomizdat, Moscow, 1980).

[9] N. D. Birrell and P. C. W. Davies, Quantum Fields in Curved Space (Cambridge University Press, Cambridge, England, 1982).

[10] C. Will, Theory and Experiment in Gravitational Physics (Cambridge University Press, Cambridge, England, 1981).

[11] L. P. Grishchuk, Phys. Rev. D 50, 7154 (1994).

[12] L. P. Grishchuk and Yu. V. Sidorov, Phys. Rev. D 42, 3413 (1990); in Quantum Gravity, edited by M. A. Markov, V. A. Berezin, and V. P. Frolov (World Scientific, Singapore, 1991), p. 678; L. P. Grishchuk, Phys. Rev. Lett. 70, 2371 (1993); in Quantum Fluctuations, edited by S. Reynaud, E. Giacobino, and J. Zinn-Justin (Elsevier Science, Amsterdam, 1997), p. 541.

[13] B. Allen, E. E. Flanagan, and M. A. Papa, Phys. Rev. D 61, 024024 (2000).

[14] L. P. Grishchuk, Phys. Rev. D 53, 6784 (1996).

[15] A. Dimitropoulos and L. P. Grishchuk, Int. J. Mod. Phys. D 11, 259 (2002).

[16] C. B. Netterfield et al., Astrophys. J. 571, 604 (2002); N. W. Halverson et al., ibid. 568, 38 (2002); R. Stompor et al., Astrophys. J. Lett. 561, L7 (2001).

[17] P. de Bernardis et al., Astrophys. J. 564, 559 (2002).
[18] S. Weinberg, Relativistic Astrophysics: 20th Texas Symposium, Austin, Texas, December, 2000, edited by J. C. Wheeler and H. Martel, AIP Conf. Proc. No. 586 (AIP, Melville, NY, 2001), pp. 893-907.

[19] S. Weinberg, Phys. Rev. D 64, 123511 (2001).

[20] S. Weinberg, Phys. Rev. D 64, 123512 (2001).

[21] F. W. J. Oliver, in Handbook of Mathematical Functions, edited by M. Abramowitz and I. A. Stegun (Dover, New York, 1964), pp. 355-433; H. A. Antosiewicz, ibid., pp. 435-477.

[22] L. P. Grishchuk, Phys. Rev. D 48, 3513 (1993).

[23] B. Allen and S. Koranda, Phys. Rev. D 50, 3713 (1994); S. Koranda, University of Wisconsin-Milwaukee thesis, 1995.

[24] R. K. Sachs and A. M. Wolfe, Astrophys. J. 147, 73 (1967).

[25] W. Hu, U. Seljak, M. White, and M. Zaldarriaga, Phys. Rev. D 57, 3290 (1998).

[26] L. P. Grishchuk, “The Early Universe Odyssey With Gravitational Waves" gr-qc/0202072.

[27] Ya. Zel'dovich and I. Novikov, Relativistic Astrophysics (The University of Chicago Press, Chicago, 1983), Vol. 2.

[28] The notion of the "moving zeros" was suggested by J. Peebles in a private correspondence, May 1990.

[29] W. Hu and S. Dodelson, "Cosmic Microwave Background Anisotropies," astro-ph/0110414.

[30] A. H. Guth and S.-Y. Pi, Phys. Rev. Lett. 49, 1110 (1982).

[31] S. W. Hawking, Phys. Lett. 115B, 295 (1982).

[32] A. A. Starobinsky, Phys. Lett. 117B, 175 (1982).

[33] J. M. Bardeen, P. J. Steinhardt, and M. S. Turner, Phys. Rev. D 28, 679 (1983).

[34] A. D. Linde, Particle Physics and Inflationary Cosmology (Gordon and Breach, New York, 1990).

[35] V. F. Mukhanov, H. A. Feldman, and R. H. Brandenberger, Phys. Rep. 215, 203 (1992).

[36] D. H. Lyth and A. Riotto, Phys. Rep. 314, 1 (1999).

[37] A. Liddle, An Introduction to Modern Cosmology (Wiley, New York, 1999).

[38] G. Efstathiou et al., "Evidence for a non-zero Lambda and a low matter density from a combined analysis of the $2 \mathrm{dF}$ Galaxy Redshift Survey and Cosmic Microwave Background Anisotropies," astro-ph/0109152.

[39] T. J. Pearson et al., "The anisotropy of the microwave background to $l=3500$ : mosaic observations with the Cosmic Background Imager," astro-ph/0205388. 